\title{
A role for the tyrosine kinase ACK1 in neurotrophin signaling and neuronal extension and branching
}

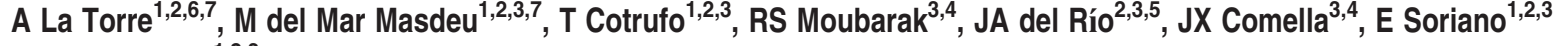 \\ and JM Ureña*,1,2,3
}

Neurotrophins are involved in many crucial cellular functions, including neurite outgrowth, synapse formation, and plasticity. Although these events have long been known, the molecular determinants underlying neuritogenesis have not been fully characterized. Ack1 (activated Cdc42-associated tyrosine kinase) is a non-receptor tyrosine kinase that is highly expressed in the brain. Here, we demonstrate that Ack1 is a molecular constituent of neurotrophin signaling cascades in neurons and PC12 cells. We report that Ack1 interacts with Trk receptors and becomes tyrosine phosphorylated and its kinase activity is increased in response to neurotrophins. Moreover, our data indicate that Ack1 acts upstream of the Akt and MAPK pathways. We show that Ack1 overexpression induces neuritic outgrowth and promotes branching in neurotrophin-treated neuronal cells, whereas the expression of Ack1 dominant negatives or short-hairpin RNAs counteract neurotrophin-stimulated differentiation. Our results identify Ack1 as a novel regulator of neurotrophin-mediated events in primary neurons and in PC12 cells.

Cell Death and Disease (2013) 4, e602; doi:10.1038/cddis.2013.99; published online 18 April 2013

Subject Category: Neuroscience

Nervous system functions require exquisitely precise neuronal circuits. The patterning and specificity of these connections are dependent on specific signals, including guidance cues and growth factors. Overall, neurons exhibit type-specific patterns of neuritic arbors that define the physiological properties of each neuronal population. Moreover, these dendritic and axonal trees are sculpted and refined by environmental signals. ${ }^{1-5}$ Neurotrophins have critical roles in neuronal differentiation. ${ }^{6-8}$ They comprise a family of structurally and functionally related proteins: nerve growth factor (NGF), brain-derived neurotrophic factor (BDNF), neurotrophin-3 (NT-3), and neurotrophin-4/5 (NT-4/5). Neurotrophin signals are transduced through Trk receptor tyrosine kinases and p75 receptor (p75NTR). ${ }^{9-11}$ The activation of Trk receptors triggers the dimerization, kinase activation, and autophosphorylation of the receptors, followed by the activation of several signaling pathways, including Ras/MAPK and PI3K/Akt.

The Activated Cdc42-associated tyrosine kinase (Ack1) is a non-receptor tyrosine kinase that was originally characterized as a target of the Rho protein Cdc42. ${ }^{12}$ The domain structure of Ack1 is unique among cytosolic kinases and contains an SH3 domain C-terminal to the kinase domain, a Cdc42/Rac interactive binding (CRIB) module, and a huge polyproline region.
In addition, Ack1 also interacts with the molecular adaptor Grb2, ${ }^{13}$ as well as with the kinases Src, Fyn, and Hck, ${ }^{14-16}$ the Ras-exchange factor Ras-GRF1, ${ }^{17} \mathrm{Dbl},{ }^{18}$ and the WiskottAldrich Syndrome Protein (WASP), ${ }^{19}$ among others.

There is evidence that Ack 1 is activated by a variety of extracellular cues, including growth factors, M3 muscarinic receptors, and chondroitin sulfate proteoglycans. ${ }^{13,14,20}$ Furthermore, Ack1 is recruited to activated RTK, such as EGFR and PDGFR. ${ }^{21,22}$ Several studies have demonstrated that Ack2 (an isoform of Ack isolated from bovine brain) coordinates changes in cell morphology required for differentiation, including the re-organization of the actin cytoskeleton. ${ }^{15,23}$ In previous studies, we showed that Ack1 is highly expressed in the central nervous system (CNS) during adulthood and in developing neurons. ${ }^{24,25}$ In neuronal cultures and in vivo, Ack1 is localized in developing dendrites and axons, including growth cones, presynaptic terminals, and dendritic spines. Here, we demonstrate that this kinase is activated by Trk signaling in neurons. To further address the cellular functions of Ack1 in response to neurotrophins, we studied the effects of Ack1 overexpression and downregulation in several neuronal models. Ack1 stimulated neuronal arborization and this effect was enhanced by neurotrophins. These findings provide evidence of the involvement of Ack1 in

\footnotetext{
${ }^{1}$ Developmental Neurobiology and Regeneration Lab, IRB Barcelona, Barcelona Science Park, Baldiri Reixac 10, 08028 Barcelona, Spain; '²epartment of Cell Biology, Faculty of Biology, University of Barcelona, Diagonal 643, 08028 Barcelona, Spain; ${ }^{3}$ Centro de Investigación Biomédica en Red para Enfermedades Neurodegenerativas (CIBERNED), Instituto de Salud Carlos III (ISCIII), Madrid, Spain; ${ }^{4}$ Cell Signaling and Apoptosis Group, Fundació Institut de Recerca Vall d'Hebron, Passeig Vall d'Hebron 119-129, Barcelona 08035, Spain and ${ }^{5}$ IBEC, Institute of Bioengineering of Catalonia, Baldiri Reixac 10, Barcelona 08028, Spain

${ }^{*}$ Corresponding author: JM Ureña, Developmental Neurobiology and Regeneration Lab, IRB Barcelona, Barcelona Science Park, Baldiri Reixac 10, Barcelona 08028, Spain. Tel: + 34 934037115; Fax: + 34 934037116; E-mail: jurena@ub.edu

${ }^{6}$ Current address: Department of Biological Structure, School of Medicine, University of Washington, Seattle, WA 98195, USA.

${ }^{7}$ These authors contributed equally to this work.

Keywords: neurotrophins; tyrosine kinase; dendritic; axonal; branching

Abbreviations: Ack1, activated Cdc42 kinase; Ack1-KD, Ack1 kinase-dead construct; Ack1-PR, Ack1 proline-rich region only construct; BDNF, brain-derived neurotrophic factor; ERK 1/2, extracellular regulated kinase 1/2; NGF, nerve growth factor; NT-3, neurotrophin-3; NT-4/5, neurotrophin-4/5; p75NTR, p75 receptor Received 19.11.12; revised 09.1.13; accepted 29.1.13; Edited by A Verkhratsky
} 
neurotrophin signaling pathways and indicate a novel role for this tyrosine kinase in neuronal differentiation.

\section{Results}

Monoclonal antibody preparation. Monoclonal antibodies were generated against a GST-Ack1 fusion protein. To characterize the immunological properties of the antibodies, we analyzed the detection of Ack1 by western blot in human embryonic kidney 293T (HEK 293T) cells transfected with Ack1 (Figure 1a) and in brain lysates immunoprecipitated with the same monoclonal antibodies (Figure 1b). Negative controls showed no band in brain lysates (Figure 1b) or in transfected HEK 293T cells (Figure 1a). Immunofluorescence of Ack1-transfected HEK 293T cells showed intracellular labeling (Figure 1c) equivalent to previously described antibodies. ${ }^{24,25}$ Two monoclonal antibodies, 23 and 25 , were found to give strong signals in western blots, immunolocalization and immunoprecipitation assays (Figures $1 \mathrm{a}-\mathrm{c}$ ).

Ack1 is tyrosine phosphorylated upon stimulation by neurotrophins. Serum-starved PC12 cells were treated for 5-60 min with NGF $(50 \mathrm{ng} / \mathrm{ml})$. Subsequently, lysates were immunoprecipitated with an anti-Ack1 antibody and analyzed by western blotting with an anti-phosphotyrosine antibody. We detected a clear increase in endogenous Ack1 phosphorylation levels upon NGF stimulation (Figure 1d) that reached maximum levels between 5 and $15 \mathrm{~min}$ post treatment (Figures 1d and e). We also observed concomitant tyrosine phosphorylation of Trk receptors immunoprecipitated using a pan-Trk antibody (Figure 1d). To establish whether other neurotrophins also stimulate neuronal Ack1, forebrain primary cultures were grown for 7 days and were then stimulated with either BDNF or NT-3. Ack1 phosphorylation levels were significantly increased after a 5-min stimulation with BDNF or NT-3 (Figures 1f-i). Again, neurotrophin treatments resulted in tyrosine phosphorylation of Trk receptors (Figures $1 \mathrm{f}$ and $\mathrm{h}$ ).

In addition, tyrosine phosphorylation was also observed when neurons were pre-treated with Src inhibitor PP2 (Supplementary Figure 1).Under these conditions, a modest increase in Ack1 phosphorylation was detected, whereas phospho-Akt was dramatically inhibited due to Src inhibition, thus indicating that Ack1 activation after neurotrophin stimuli is not dependent on Src activity (Supplementary Figure 1).

To ascertain whether neurotrophins regulate Ack1 kinase activity, PC12 cells and cortical neurons were treated with neurotrophins (NGF, BDNF, NT-3). This treatment produced a modest but significant increase in Ack1 kinase activity (Figure 1j), consistent with the increase in phosphorylation levels previously detected. In addition, transfected HEK 293T cells showed a high increase in the kinase activity of Ack1 as a result of its overexpression. Such an increase was not observed in constructs lacking kinase domain or with a mutation in the active site (Figure $1 \mathrm{k}$ ) with similar levels (Supplementary Figure 2). We thus concluded that members of the neurotrophin family lead to Ack1 tyrosine phosphorylation and kinase activation.
Ack1 interacts with Trk receptors but not with $\mathrm{p} 75^{\mathrm{NTR}}$. To address the role of Ack1 in neurotrophic receptor signaling pathways, we studied the interaction between Ack1 and Trk receptors, and Ack1 and $\mathrm{p} 75^{\mathrm{NTR}}$. For this purpose, HEK 293T cells were co-transfected with hemagglutinin (HA)-tagged Ack $1,{ }^{25}$ and one of the following cDNAs: TrkA, GFP-tagged TrkB, ${ }^{26}$ Myc-tagged $\operatorname{TrkC}^{27}$ or $\mathrm{p} 75^{\mathrm{NTR}}$ constructs $^{28}$ (Figure 2).

Lysates from HEK 293T cells transfected either with an empty vector as a control or with Ack1, TrkA or with both constructs simultaneously (Figure $2 \mathrm{a}$ ), and immunoprecipitated with anti-HA or with anti-TrkA antibody revealed TrkA after immunoprecipitation with anti-HA (upper panel, fourth lane) as well as Ack1 after Trk immunoprecipitation (third panel, fourth lane). When cells co-transfected with plasmids coding for Ack1-HA and GFP-TrkB were immunoprecipitated with either anti-HA or anti-GFP antibodies, the corresponding partner was detected by western blot (Figure 2b). Similar results were obtained when we co-transfected HEK 293T cells with TrkC-Myc and Ack1-HA (Figure 2c). Cell lysates were immunoprecipitated either with anti-Myc or with anti-HA antibodies and the reciprocal interacting protein in the resulting immunoprecipitates was detected by western blot (Figure 2c). However, when the same experiments were performed with plasmids corresponding to $\mathrm{p} 75^{\mathrm{NTR}}$ and Ack1$\mathrm{HA}$, the complementary protein was not detected (Figure 2d). These results indicate that Ack1 co-immunoprecipitates with Trk receptors but not with the p75 ${ }^{\mathrm{NTR}}$ receptor.

We performed Ack1/TrkB immunoprecipitations in brain lysates from embryonic E16 brains. Here, we observed co-immunoprecipitation between the two proteins when we immunoprecipitated Ack1 (Figure 2e) and TrkB (Figure 2f).

NGF leads to a subcellular redistribution of Ack1 in PC12 cells. In developing and adult neurons, Ack1 shows a cytoplasmic distribution, ${ }^{24,25}$ while in some cell lines it shows a punctuate pattern in the cell cytoplasm and perinuclear regions. ${ }^{21}$ Given the observation of the interaction between neurotrophin receptors and Ack1, we applied immunolabeling to analyze whether neurotrophin treatments cause a redistribution of Ack1 expression in PC12 cells. While starved cells showed the expected punctuated expression pattern (Figure 3a), short stimulations with $50 \mathrm{ng} / \mathrm{ml}$ of NGF (5 min) induced a clear re-arrangement of Ack1 protein distribution, which was reflected by the presence of Ack1 aggregates in the outer edges of the cells in $>60 \%$ of the analyzed cells (Figures $3 \mathrm{~b}$ and $\mathrm{c}$ ). In these conditions, Ack1 immunostaining greatly co-localized with NGF (Figures $3 d-i)$. Furthermore, stimulation of PC12 cells with NGF led to strong co-localization of Ack1 and TrkA, which formed clusters located near the cell surface (Figures 3j-l).

In addition, we performed co-localization experiments with filamental actin (F-actin). In control PC12 cells, a significant part of the Ack1 protein showed partial co-localization with F-actin, which was present mainly in perinuclear areas (Figures 3m-0). Interestingly, when PC12 cells were incubated with NGF, co-localization of Ack1 and F-actin was also detected in protruding tips at the cell surface, as demonstrated by Phalloidin-FITC co-staining with anti-Ack1 antibody (Figures 3p-u). The re-arrangement of Ack1 protein 


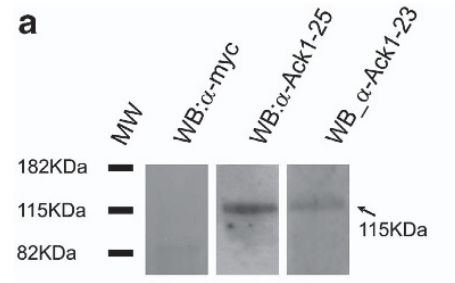

b

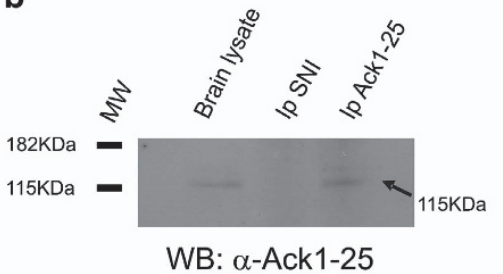

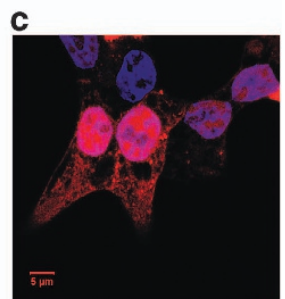

e

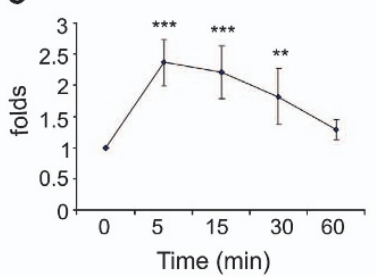

g

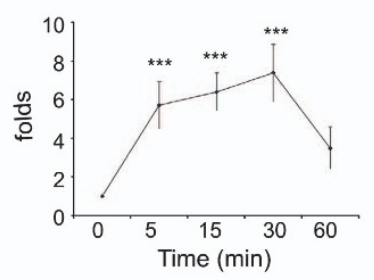

i
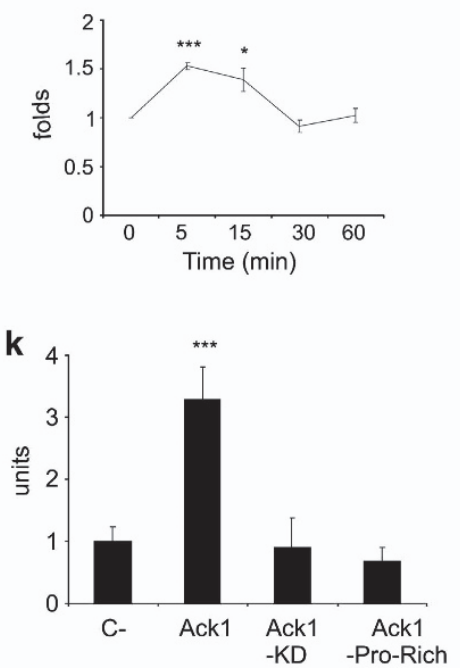

Figure 1 Neurotrophin-dependent phosphorylation of Ack1 protein. Monoclonal antibodies against Ack1 show detection by western blot in HEK 293T cells overexpressing Ack1 (a), and by co-immunoprecipitation and western blot in brain lysates (b). In addition, immunofluorescence with the antibodies in Ack1-transfected HEK 293 cells shows differential labeling in the transfected cells compared with non-transfected ones (c) using antibody 25 . For immunoprecipitation experiments, $20 \%$ of the input was loaded into the gel. PC12 (d and e) and neuronal cultures ( $\mathbf{f}-\mathbf{i}$ ) were treated for $5,15,30$, and 60 min with $50 \mathrm{ng} / \mathrm{ml}$ of NGF (d and e), BDNF ( $\mathbf{f}$ and $\mathbf{g}$ ), or NT-3 (h and i). (d, $\mathbf{f}$, and $\mathbf{~ h}$ ) Western blot analyses of phospho-Ack1 (upper panels) and phospho-Trk (third panels) and their loading controls are shown. Neurotrophin treatments induced tyrosine phosphorylation of Ack1 and Trk receptors. Ack1 activation was observed from 5 to $15 \mathrm{~min}$. (e, $\mathbf{g}$, and i) Densitometric analyses of 5-7 independent experiments normalized to untreated samples $(t=0)$ showing neurotrophin-dependent Ack1 phosphorylation are represented. Each point is represented as mean $\pm S$.E.M. $\left({ }^{* \star} P<0.05 ;{ }^{* \star *} P<0.001\right)$. Each group was compared with their corresponding control at $t=0$ by $T$-test. (j) In vitro kinase assay shows activation of Ack 1 by neurotrophin treatment in PC12 cells (NGF dependent), and primary hippocampal neurons (BDNF and NT-3 responsive). (j) Groups were compared with their corresponding control at time $0^{\prime}$. (k) In transfected HEK 293T cells, we detected clear activation in Ack1-overexpressing cells and basal levels of activation in Ack1-KD- or Ack1-PR-overexpressing cells. Each group was compared with control cells using T-test $\left({ }^{* *} P<0.05 ;{ }^{* \star *} P<0.001\right)$

distribution after stimulation with NGF suggests that part of the activated pool of Ack1 is detected close to NGF-activated regions, which might result in protruding edges.
PC12 cells overexpressing Ack1 show increased neuritic outgrowth. To elucidate whether Ack1 affects neuronal differentiation, we established PC12 cell lines 

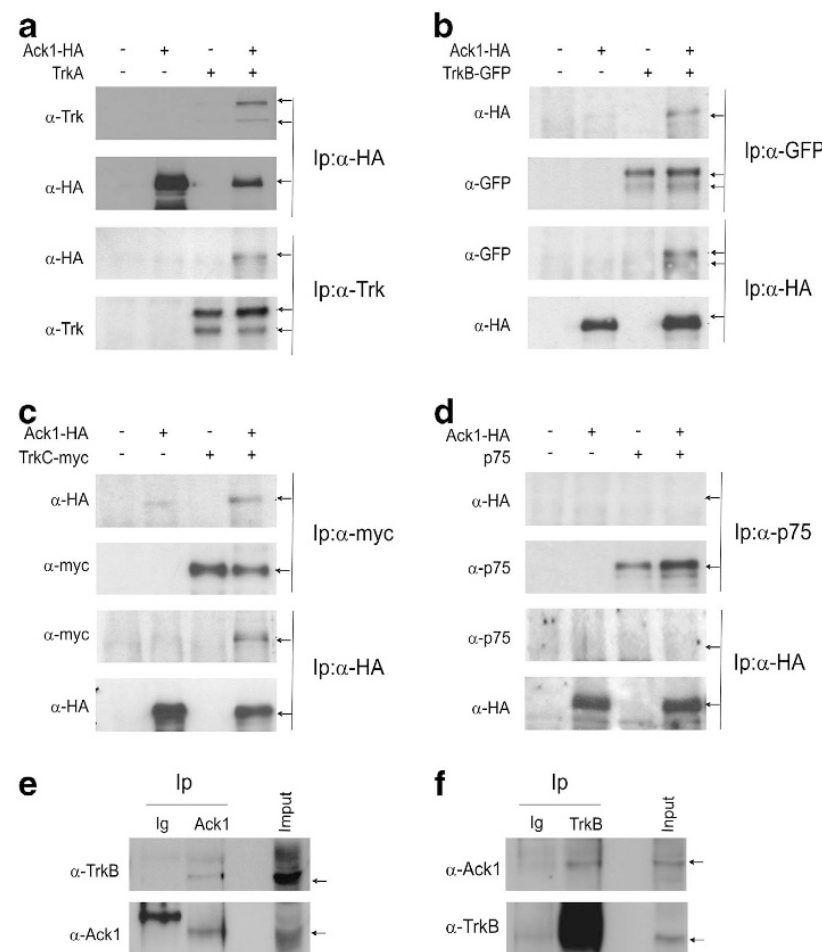

Figure 2 Ack1 and Trk receptors interact both in transfected HEK 293T cells and in brain lysates. HEK 293T cells were transiently transfected with Ack1-HA and TrkA (a), TrkB-GFP (b), TrkC-myc (c), or p75 constructs (d). After immunoprecipitation, we detected the corresponding partner when Ack1 and Trk receptors were co-expressed (a-c, lane 4), but p75 and Ack1 failed to show any association (d, lane 4). Brain lysates from mouse embryos (E16) were subjected to immunoprecipitation with anti-Ack1, anti-TrkB (Upstate), or anti-GFP as control. Endogenous Ack1 and Trk receptors interacted in the developing brain (e and $\mathbf{f}$, lane 2)

stably overexpressing the wild-type Ack1 cDNA. PC12 cells have been extensively used as a model of differentiation. ${ }^{29,30}$ Two clones (named as PC12-Ack\#1 and Ack\#2) were obtained and presented increased intracellular levels of Ack1 protein (about 3-5 times), in comparison with control cells by western blot assays (Figures $4 \mathrm{~b}$ and c). Interestingly, a considerable proportion of Ack1-overexpressing PC12 cells displayed a flattened phenotype and extended neurites even in NGF-free medium (Figure 4a), and the percentage of differentiated cells increased with time (Figure 4d).

To study whether Ack1 influenced NGF-dependent neurite growth, time-course experiments were performed. Ack1overexpressing cells responded to NGF, and the fraction of cells with neurites was significantly higher at all time points compared with control cells (PC12 or PC12-pRK5, Figures 4e and $\mathrm{f}$ ), as was the total neuritic arbor length (Figure $4 \mathrm{~g}$ ). Interestingly, we also observed an increased number of branching points in PC12-Ack1 cells, which was especially evident at 10 DIV (Figures $4 \mathrm{e}$ and $\mathrm{h}$ ). These data indicate that Ack1 overexpression increases neuronal differentiation and neurite complexity in NGF-stimulated and unstimulated PC12 cells.

Endogenous Ack1 protein influences NGF-induced neuritic outgrowth in PC12 cells. Ack1 overexpression led to the appearance of numerous neurites, and NGF enhanced this effect. We hypothesized that Ack1 is involved in neurotrophin-mediated differentiation in PC12 cells. To evaluate the role of endogenous Ack1 in neurite-outgrowth events, we used several RNAi constructs that target distinct sites of the Ack1 sequence (Sigma's Mission TRC shRNA lentiviral-based constructs, Sigma). We generated stably transfected PC12 cell lines with short-hairpin RNA (shRNA)expressing vectors and analyzed the capacity of each RNAi to knock down endogenous Ack1 expression in PC12 cells. Two of these cell lines showed a dramatic decrease in Ack1 protein expression (Figures $4 \mathrm{j}$ and $\mathrm{k}$ ) compared with wildtype PC12 cells or with control RNAi (scrambled-shRNA), as demonstrated by western blot analysis. To study the effect of Ack1 silencing on NGF-induced differentiation, PC12 cell lines were treated with $50 \mathrm{ng} / \mathrm{ml}$ of $\mathrm{NGF}$ for several days, and the neuritic processes were monitored. RNAi-mediated knock-down of Ack1 partially counteracted NGF-induced outgrowth after 5-10 days of treatment (Figures 4i, I, and $\mathrm{m}$ ). Both the percentage of differentiated cells and the number of branching points were significantly decreased in RNAi-PC12 cells (Figures $4 \mathrm{I}$ and $\mathrm{m}$ ). Similar effects were observed with the two RNAi sequences. We checked that these cell lines did not show affected viability (Supplementary Figure 3).

To exclude off-target effects of the shRNA sequences, we performed rescue experiments with a shRNA-resistant construct of Ack1. This construct was generated by mutating the nucleotide sequence that targets the shRNA of Ack1 but maintaining the amino-acid sequence of the protein. We used PC12 cell lines expressing RNAi-mediated Ack1 knock-down and transfected them with the shRNA-resistant Ack1. This construct effectively counteracted the effect of RNAi and showed a more differentiated phenotype than in the PC12RNAi cells (Supplementary Figure 4).

Ack1 signaling modulates the PI3K and MAPK pathways in response to neurotrophin stimulation. We analyzed NGF-activated pathways in the PC12 cell lines in time-course experiments, in which the cells were treated with $50 \mathrm{ng} / \mathrm{ml}$ of NGF for 5, 15, or $30 \mathrm{~min}$. Western blotting was used to assess the extent of Akt and extracellular regulated kinase 1/2 (ERK 1/2) phosphorylation. In Ack1-overexpressing cells, Akt phosphorylation in response to neurotrophins was significantly higher and persisted for longer than in control cells (Figures $5 \mathrm{a}$ and c). Conversely, analyses of PC12-RNAi cells showed that the peak of Akt1 phosphorylation occurred after 5-15 $\mathrm{min}$, but these increases were smaller than those observed in control cells (Figures $5 b$ and $c$ ) ( $P$ lower than 0.001 at $15 \mathrm{~min})$.

Similarly, phospho-Erk 1/2 levels were higher in PC12-Ack1 cells after 5 min of NGF treatment and at 15-30 min increased phosphorylation levels were still detected (Figures $5 a$ and d). Moreover, Ack1 knock-downs resulted in a marked decrease in the ERK $1 / 2$ phosphorylation levels at 5-15 min (Figures $5 \mathrm{~b}$ and d). In addition, pharmacological blockade of the PI3K or MAPK pathways did not affect the neurotrophin-induced phosphorylation of Ack1, whereas it did block phospho-Akt1 and phospho-Erk $1 / 2$ levels. In a similar manner, this pharmacological blockade does not affect the neurotrophininduced increase in kinase activity of Ack1 (Supplementary Figure 5). Taken together, these data suggest that Ack1 

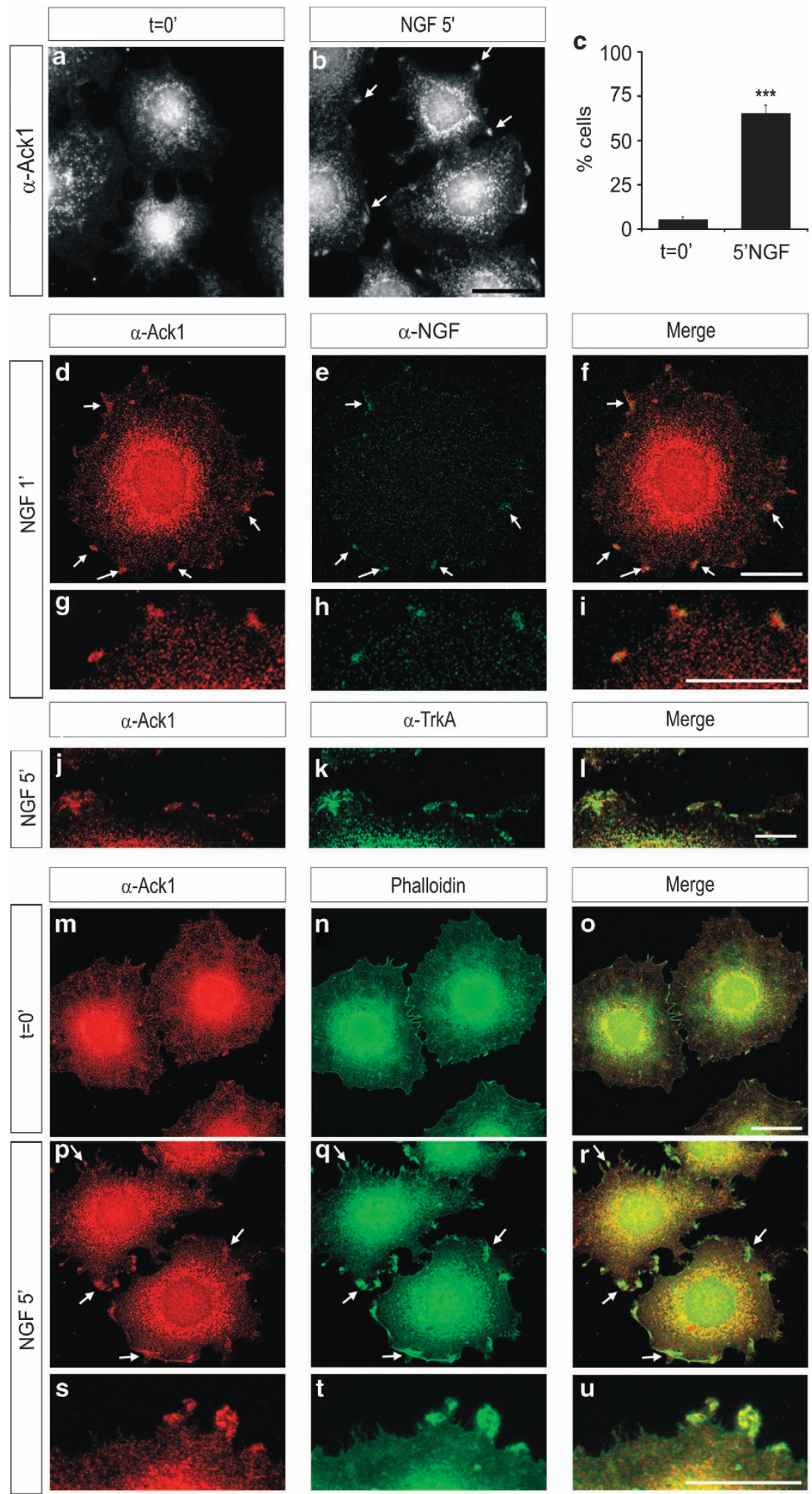

Figure 3 Neurotrophin treatment causes a re-organization of Ack1 in PC12 cells. Immunostaining analyses of PC12 cells showing the distribution of Ack1 in untreated cells (a) and after NGF treatments (b). NGF stimuli promoted a redistribution of Ack1 protein, which is reflected in the presence of Ack1 in the cell edge (arrows). This re-organization of Ack1 was detectable in $>60 \%$ of the cells (c). (d-i) Co-localization between Ack1 and NGF in PC12 cells after short stimulations with NGF. PC12 cells were incubated with anti-Ack1 and anti-NGF. As previously described, a significant pool of Ack1 protein was detected in the cell membrane and these Ack1 aggregates greatly co-localized with NGF. (j-I) Co-localization between Ack1 and TrkA in PC12 cells after a 5-min stimulation with NGF. As previously shown, a significant amount of Ack1 protein was co-localized with TrkA in cell membrane clusters. Also, Ack1 significantly co-localized with F-actin in these discrete membrane edge regions ( $\mathbf{m}-\mathbf{u})$. Scale bars: $25 \mu \mathrm{m}$ (a and $\mathbf{b}), 20 \mu \mathrm{m}$ (d-f and $\mathbf{m}-\mathbf{r})$, and $10 \mu \mathrm{m}(\mathbf{g}-\mathbf{I}$ and $\mathbf{s}-\mathbf{u})$ 
activation is upstream of the PI3K and MAPK pathways and that Ack 1 protein levels regulate Akt1 and ERK 1/2 responses to neurotrophins.
To ascertain whether the activation of Akt and ERK 1/2 can be due, at least in part through Ras activation, we performed Ras assay with different construct of Ack1: full-length (Ack1), a

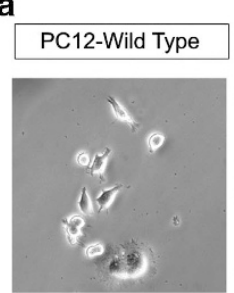

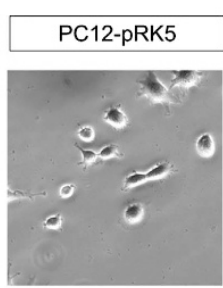

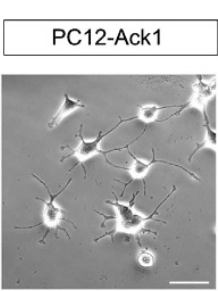

b

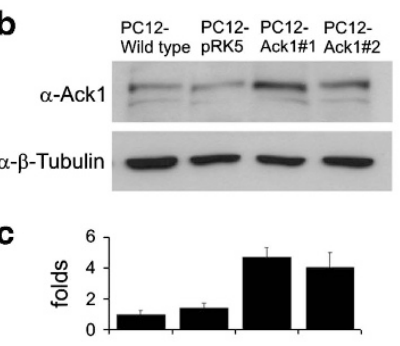

d

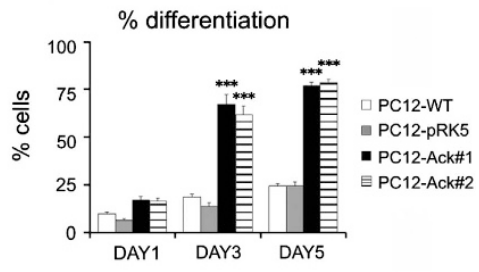

e
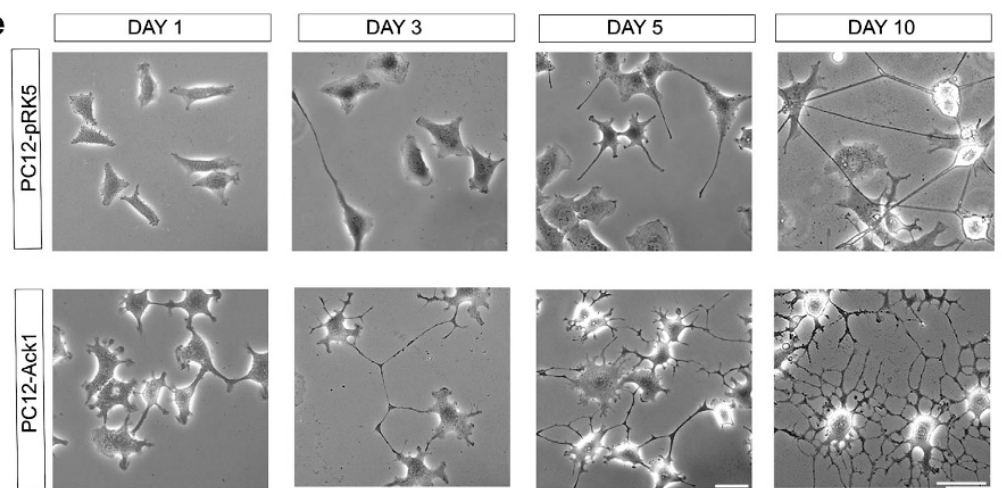

f

$\%$ differentiation

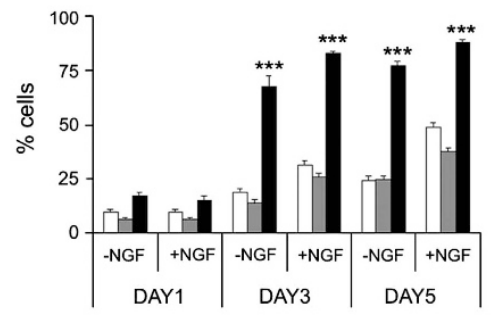

$\square$ PC12-WT $\square$ PC12-pRK5 $\square$ PC12-Ack1

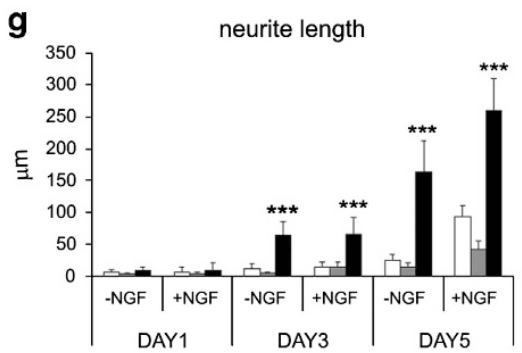

h branching points

$\square$ PC12-WT $\square$ PC12-pRK5 —PC12-Ack1
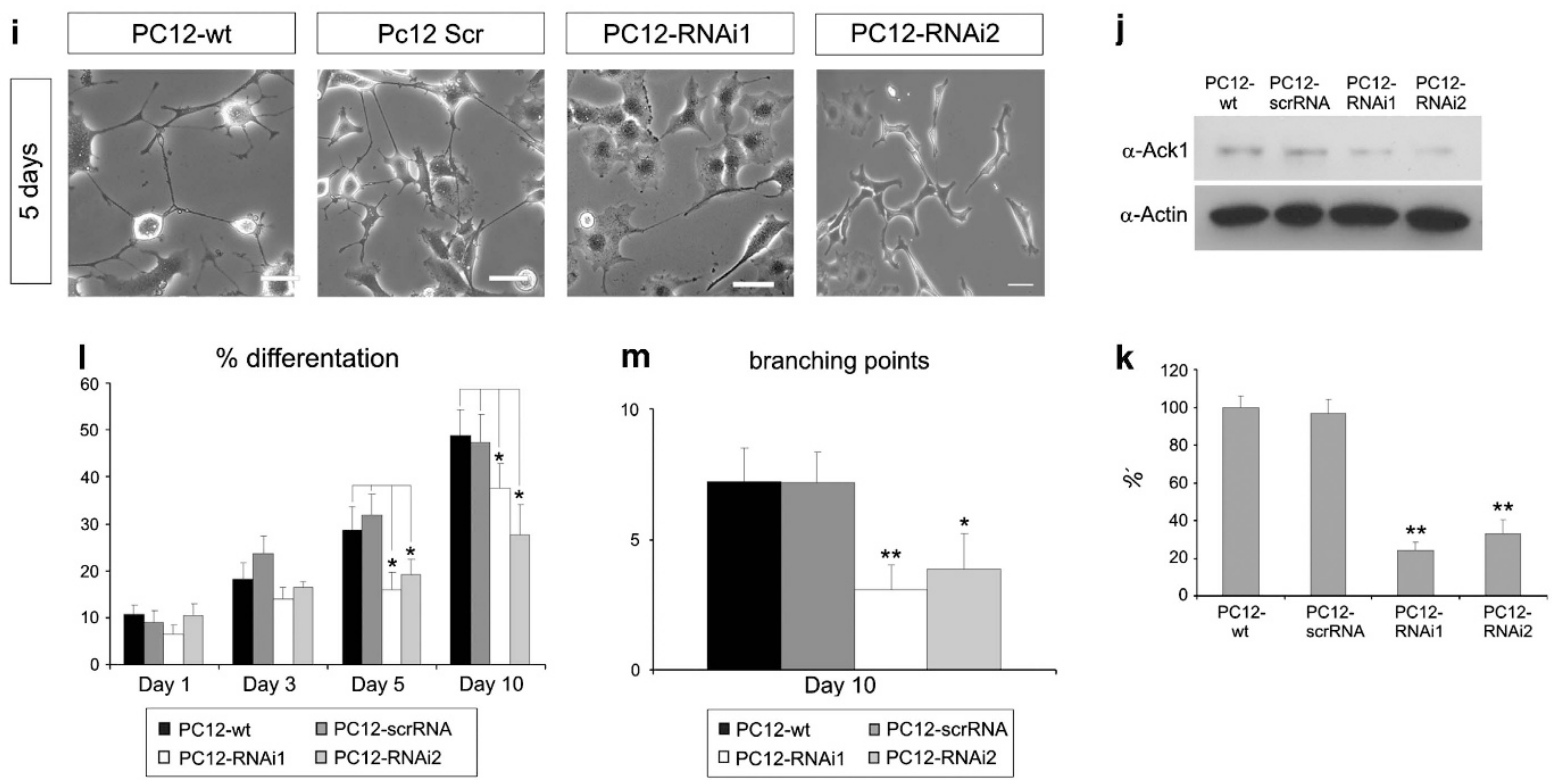
a

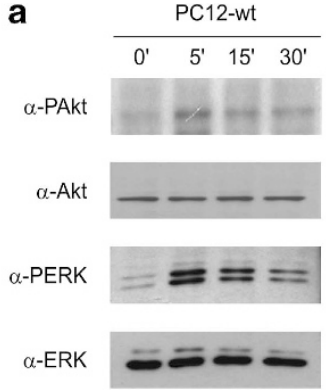

b

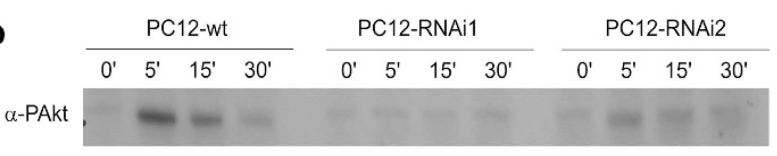

$\alpha-A k t$
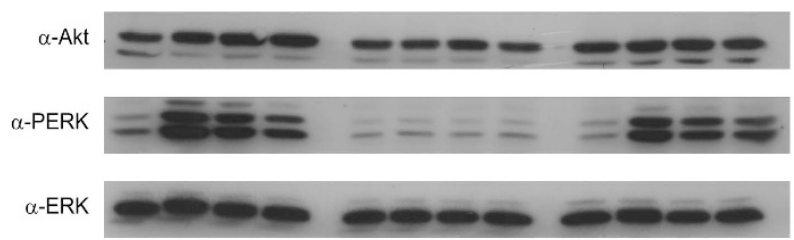

c

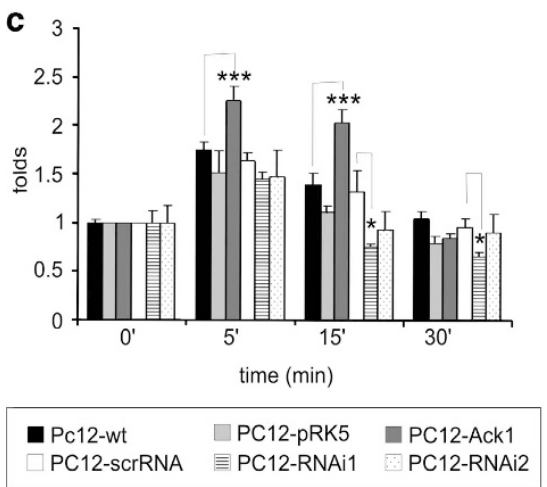

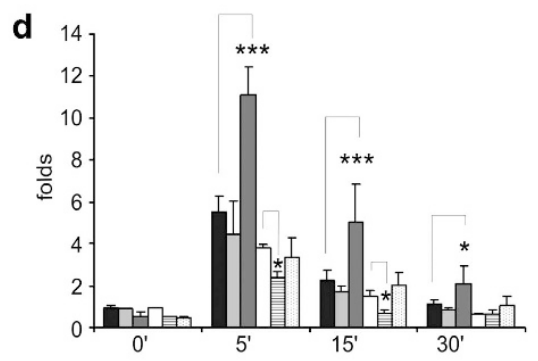

time (min)

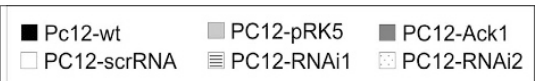

Figure 5 Ack1 modulates Akt1 and MAPK pathways. (a) Starved PC12 cells lines (wild-type PC12 cells, lanes 1-4, PC12 cells stably transfected with an empty vector, lanes 5-8; PC12 cells overexpressing Ack1i, lanes 9-12) were treated for 5, 15, 30, and 60 min with NGF (50 ng/ml) or left untreated ( $t=0)$ (lanes 1, 5, and 9). Overexpression of Ack1 enhanced the NGF-induced phosphorylation of Akt (panel 1, lanes 9-12) and ERK 1/2 (panel 3, lanes 9-12). Loading controls are in panels 2 and 4. (b) Starved PC12 cells lines (PC12 wild type, lanes 1-4; PC12 expressing two shRNAs against Ack1, lanes 5-12) were treated for 5, 15, 30, and 60 min with NGF (50 ng/ml) or left untreated $(t=0)$ (lanes 1, 5, and 9). Ack1 knock-down counteracted the NGF-induced phosphorylation of Akt (panel 1, lanes 5-8 and 9-12) and ERK 1/2 (panel 3, lanes 5-8 and 9-12). Loading controls are in panels 2 and 4. Quantification of NGF-induced phospho-Akt levels (c) and phospho-ERK (d) is illustrated. The mean data \pm S.E.M. of 3-6 separate experiments are shown. Data were normalized to control $t=0$. Groups compared with the $T$-test are indicated $\left({ }^{\star} P<0.01 ;{ }^{* \star *} P<0.001\right)$

Figure 4 Ack1 promotes differentiation in PC12 cells and this effect is enhanced by neurotrophins. (a) Phase-contrast morphology of PC12, PC12-pRK5 (empty vector), and PC12-Ack1 cells at 3 days of culture in NGF-free medium. Scale bar: $50 \mu \mathrm{m}$. (b) Western blot analysis of Ack1 (top) and tubulin (bottom) in non-transfected PC12 cells (wild type), PC12 cells transfected with an empty vector (PC12-pRK5), and Ack1-overexpressing cells (Ack1\#1 and Ack1\#2) (lanes 3 and 4). (c) Densitometric analysis of western blot shows an increase in the immunoreactivity of PC12-Ack1 cells to Ack1 antibody. (d) Effects of Ack1 overexpression on cellular differentiation. PC12 lines were plated and grown for the times indicated, and neurite outgrowth was analyzed. The number of differentiated cells is markedly increased upon Ack1 overexpression. Data are represented as mean \pm S.E.M. Each group is compared with its corresponding control at day 1 of treatment $\left({ }^{* * *} P<0.001\right)$. (e-h) Ack1 overexpression exerts a differentiation effect upon NGF treatments. PC12 lines were plated and grown in medium containing $50 \mathrm{ng} / \mathrm{ml}$ of NGF for the times indicated. The number of differentiated cells (f) and the total neuritic length (g) was strikingly increased upon Ack1 overexpression. Phase-contrast morphology of PC12, PC12-pRK5 (empty vector), and PC12-Ack1 cells at several days of culture is shown in (e). Scale bar: $50 \mu \mathrm{m}$. At day 10 in NGF medium, control-PC12 and PC12-Ack1 cells had distinct morphologies, as illustrated in phase-contrast micrographs (e). Ack1 overexpression induced a prominent increase in the number of branching points $(\mathbf{h})$ and the neurites were significantly shorter. Data are represented as mean \pm S.E.M. ( $\left.{ }^{\star *} P<0.05 ;{ }^{* \star \star} P<0.001\right)$. Each group was compared with its corresponding control at day 1 . In (f-h), PC12-Ack1 and PC12-pRK5 (empty vector) are compared with PC12 wild-type cells. (j) Western blot analysis showing that Ack1 RNAi (shRNA) reduces the expression of endogenous Ack1. The expression of Ack1 was normalized by the analysis of actin expression (bottom panel). (k) Densitometric analysis of western blots reveal a reduction of $74 \%$ in one case and $67 \%$ in a second case in the immunoreactivity to Ack1 antibody in the PC12-shRNA cells compared with scrambled-RNAi ones. (i, I, and $\mathbf{m}$ ) Depletion of endogenous Ack1 partially counteracted NGFinduced differentiation. (i) Phase-contrast micrographs of PC12 cells stably transfected with scrambled RNAi, or two shRNAs against Ack1 illustrate the morphology of these lines after culture in NGF for 5 days in comparison with wild-type PC12 cells. Knock-down of endogenous Ack1 inhibited the neuritic differentiation phenotype and neuritic arborization (I) promoted by NGF treatment for different periods. ( $m$ ) Quantification of the number of branching points of PC12, PC12-scrambled RNAi, and PC12-RNAi lines submitted to NGF treatment for 10 days. PC12-RNAi1 and PC12-RNAi-2 cells were compared with PC12-scrambled using the $T$-test $\left({ }^{*} P<0.01 ;{ }^{* \star} P<0.05\right)$. ( ${ }^{* \star} P<0.05$; $\left.{ }^{* \star *} P<0.001\right)$. Scale bar: $50 \mu \mathrm{m}$ 
kinase hypoactive (Ack1-KD), and proline rich (Ack1-PR). We performed this assay in PC12 cells and we observed (Supplementary Figure 6) an increase in Ras active form (Ras-GTP) after NGF stimulation, which was remarkably lower when the construct was lacking the Ack1 kinase (Ack1-PR) domain or was mutated in the active site (Ack1-KD).

Ack1 regulates dendritic and axonal branching in neurons in both BDNF-dependent and -independent manners. We studied the effects of Ack1 overexpression in neuronal branching. For this purpose, we used pyramidal hippocampal neurons, a neuronal population whose neuritic growth and branching is dependent on neurotrophin actions. $^{31-33}$ Thus, using primary hippocampal cultures, we examined whether the Ack1-regulated neuronal differentiation observed in PC12 cells is a general mechanism for neurotrophin-induced arborization. At day 4 in vitro, primary hippocampal neurons were transiently co-transfected with the pEGFP-N3 vector together with an empty vector, Ack1-FL cDNA, a kinase-dead construct (Ack1-KD), or an Ack1proline-rich region construct (Ack1-PR) lacking the kinase domain (as a dominant negative). After $24 \mathrm{~h}$, the transfected neurons were starved in control medium or treated with BDNF (dosages: 5 and $20 \mathrm{ng} / \mathrm{ml}$ ). The neurons were fixed $48 \mathrm{~h}$ later. Hippocampal neurons were stained with anti-GFP and also identified by neuronal morphology. First, we demonstrated that the Ack1-KD and -PR constructs have decreased kinase activity compared with Ack1-FL (Figure 1k), and that transfection of these constructs in $\mathrm{PC} 12$ cells prevented $\mathrm{P}$-ERK activation by neurotrophins (Figures $6 \mathrm{a}-\mathrm{r}$ ).

Transfections with Ack1-FL construct dramatically increased the number of branching points and therefore the complexity of neuritic arbors in dendrites and axons of hippocampal neurons, compared with GFP-transfected cells (Figures 7a, b, g, h, e, and k). Furthermore, this phenomenon was greatly enhanced by BDNF stimuli, similarly to Ack1overexpression effect previously described in PC12 cells (Figures 7e and k). Interestingly, transfection with Ack1-PR, a construct that lacks the entire kinase domain, blocked increases in neurite branching (in the presence and in the absence of BDNF), whereas transfection with the Ack1-K158A construct resulted in intermediate effects (Figures 7c-e and i-k).

We next measured total dendritic and axonal lengths (Figures $7 f$ and I). The effects of the Ack1 constructs on axonal length were identical to those described above for neurite branching, both in untreated neurons and in cells incubated with BDNF (Figure 7f). In contrast, total dendritic length of hippocampal neurons was significantly increased by the Ack1 cDNA in control cultures and in neurons treated with high BDNF concentrations $(20 \mathrm{ng} / \mathrm{ml})$, but not in those incubated with moderate BDNF concentrations $(5 \mathrm{ng} / \mathrm{ml})$ (Figure 7I).

To corroborate these findings, we next used P5 cerebellar granule neurons to study the influence of endogenous Ack1 on the BDNF-induced differentiation in neurons. In this neuronal type, transfection of several Ack1 constructs (Ack1, Ack1-K158A, and Ack1-PR cDNAs) gave results similar to those described above for hippocampal neurons (Figures 8a-h and $\mathrm{m}-\mathrm{p}$ ).
To further confirm these observations, we performed knock-down experiments in EGL neurons using shRNA constructs (Figures $8 \mathrm{i}-\mathrm{I}$ and $\mathrm{q}-\mathrm{r}$ ). First, we corroborated that the shRNA sequences designed effectively decreased endogenous Ack1 protein levels in neurons (Figures 6s-z). Knock-down of endogenous Ack1 did not affect branching parameters in unstimulated control neurons; however, it did result in a decrease in both dendritic and axonal branching upon BDNF incubations. This decrease was statistically significant at high BDNF concentrations (20 ng) (Figures 8i$\mathrm{I}, \mathrm{q}$, and $\mathrm{r}$ ). Taken together with the results obtained in PC12 cells, we conclude that endogenous Ack1 protein levels modulate neurotrophin-induced branching.

\section{Discussion}

Ack1 is expressed mainly in the CNS and high levels of Ack1 protein are detected in developing dendrites and axons. ${ }^{24,25}$ Although some relevant protein-protein interactions have been reported, the signaling network in which Ack1 operates has been described only in part. Ack1 has been shown to interact with many signaling molecules, including receptor tyrosine kinases and molecular adaptors such as Grb2 and Nck. ${ }^{13,21}$ Ack1 also associates with actin cytoskeleton regulators like Cdc42 $2^{12,34}$ and WASP. ${ }^{19}$ Interestingly, Ack1 also binds to Ras-GRF1, a Trk-interacting molecule related to neuronal differentiation. ${ }^{17,35,36}$ Furthermore, Ack2 overexpression promotes cellular differentiation in NIH3T3 cells ${ }^{15}$ and Ack2 molecules direct the formation of membrane protrusions in transformed cells lines. ${ }^{37}$ In addition, the Drosophila homolog of Ack1 regulates axonal guidance by the phosphorylation of a WASP-binding partner, the sorting nexin DSH3PX1. ${ }^{38}$ All these findings suggest that Ack1 is involved in the regulation of several cytoskeletal and transduction pathways that ultimately lead to neuronal differentiation. Therefore, the purpose of the present study was to analyze the pathways regulated by Ack 1 and elucidate the contribution of this kinase to the neuronal differentiation and polarization processes.

Here, we provide evidence suggesting that Ack1 has a relevant role in neurotrophin signaling pathways during neuronal polarization. We demonstrate that Ack1 is tyrosine phosphorylated in response to all the neurotrophic factors studied, thereby supporting the notion of a general role for this kinase in neurotrophin transduction pathways. Moreover, our results show that the neurotrophin receptors Trk and Ack1 interact whereas p75NTR does not bind to Ack1. These observations lead us to propose that Ack1 is involved in Trk signaling events. We also show the influence of Ack1 on functional processes triggered by neurotrophins such as differentiation in PC12 cells.

In addition, Ack1 overexpression induced neuritic arborization in all the models tested. In the same regard, the expression of dominant-negative constructs or knock-down experiments resulted in a decrease in neurite differentiation and elaboration.

We found that the proline-rich $\mathrm{C}$-terminus region is essential for coupling to Trk receptor signaling molecules and for mediating the biological activities of Ack1. Therefore, overexpression of the sole proline-rich region acts as a 

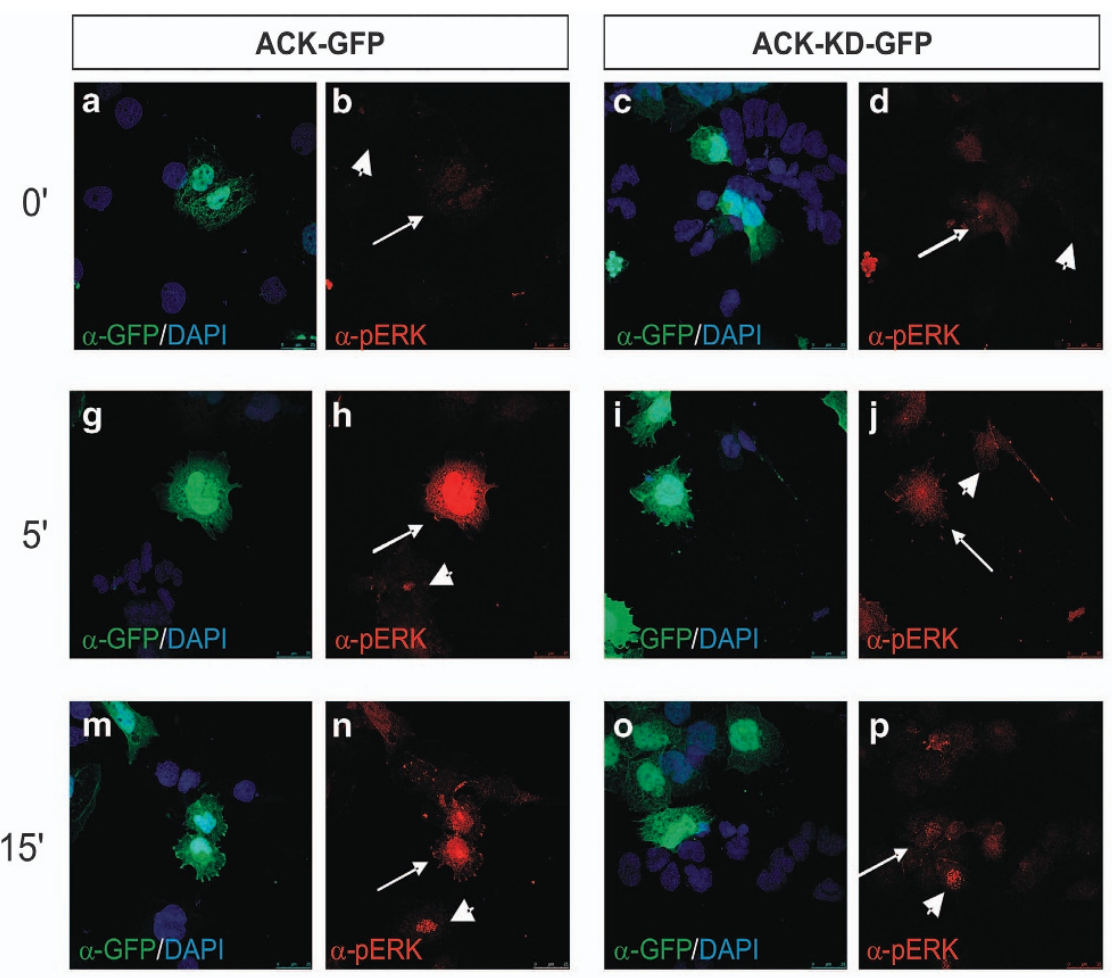
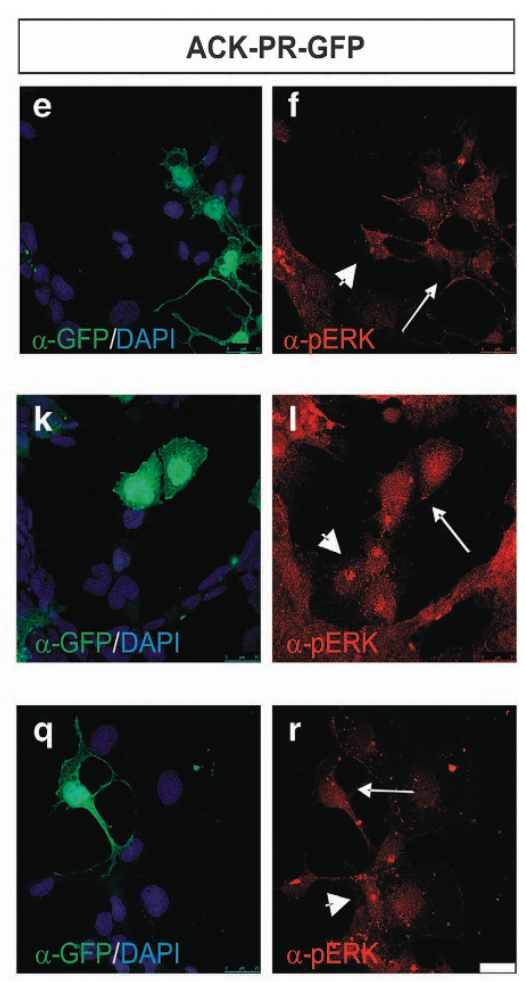
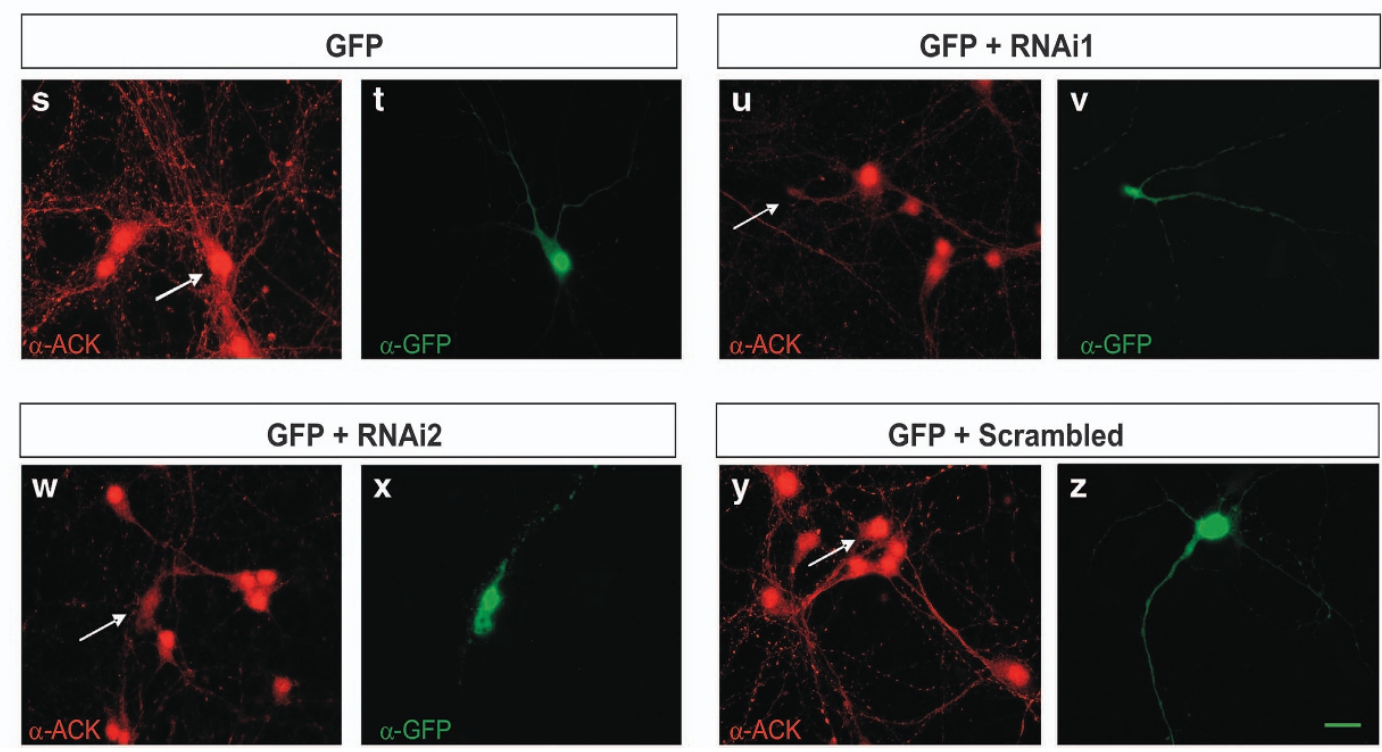

Figure 6 NGF triggers P-ERK immunolabeling in cells overexpressing Ack1 and shRNA sequences downregulate endogenous Ack1 levels in neurons. (a-r) Ack1 kinasedead and Ack1 proline-rich constructs counteracted ERK activation induced by NGF at $0^{\prime}, 5^{\prime}$, and $15^{\prime}$. PC12 cells were transfected with either pRK5-Ack1 or pRK5-Ack1 KD or pRK5-Ack1-PR and pAcEGFP vectors and labeled with anti-GFP and anti-P-ERK antibodies. Large arrows show cells overexpressing Ack1 and short arrows untransfected cells. Green fluorescence shows cells positive for Ack1 and red fluorescence cells positive for P-ERK. While cells expressing Ack1 presented activated ERK ( $\mathbf{g}, \mathbf{h}, \mathbf{m}$, and $\mathbf{n})$, those expressing either Ack1-KD (i, j, o, and p) or Ack1 PR (k, l, q, and r) showed lower activation of ERK. Scale bars: $25 \mu \mathrm{m}$ (s-z). Neurons transfected with shRNA for Ack1 presented lower Ack1 expression. Hippocampal neurons were transfected with PAc-EGFP ( $\mathbf{s}$ and $\mathbf{t}$ ) and two shRNAs for Ack1 ( $\mathbf{u}, \mathbf{v}, \mathbf{w}$, and $\mathbf{x}$ ) or a scrambled sequence of shRNA ( $\mathbf{y}$ and $\mathbf{z}$ ). Cells were stained with anti-Ack1 antibodies (s, $\mathbf{u}, \mathbf{w}$, and $\mathbf{y})$ or anti-GFP (t, $\mathbf{v}, \mathbf{x}$, and $\mathbf{z}$ ). Arrows show cells expressing Ack1 and GFP. Scale bars: $10 \mu \mathrm{m}$

dominant negative. Consistent with our data, previous studies report that the biological functions of Ack1 molecules require interactions mediated through the proline-rich domain. ${ }^{16,21,39}$ In conclusion, our results have identified a novel function of Ack1 protein in the mediation of neurite outgrowth and branching in neuronal cells, and indicate that Ack1 protein is a novel component of the Trk signaling cascade.

ERK 1/2 and Akt1 phosphorylation has a pivotal role in promoting neurite outgrowth in response to neurotrophins. ${ }^{40}$ Our biochemical data demonstrate that Ack1 signaling 

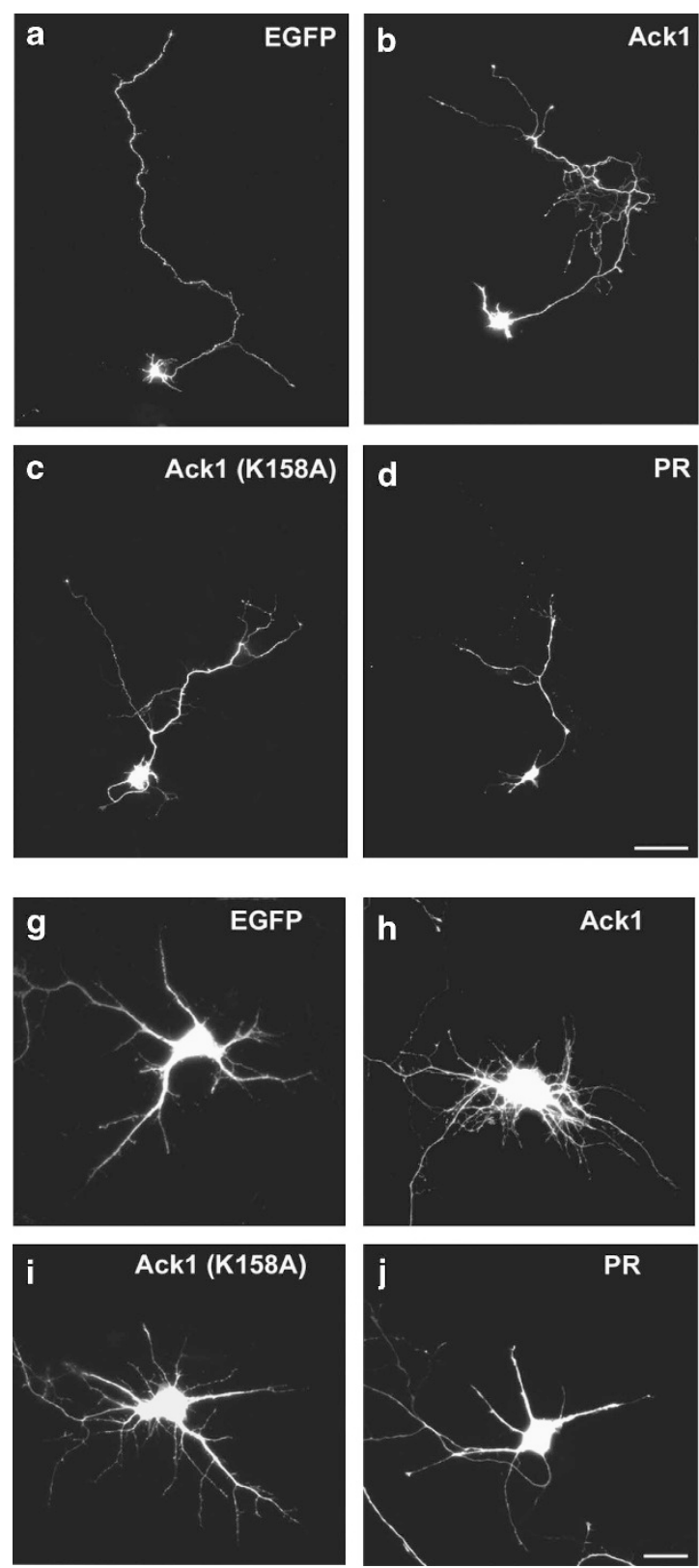
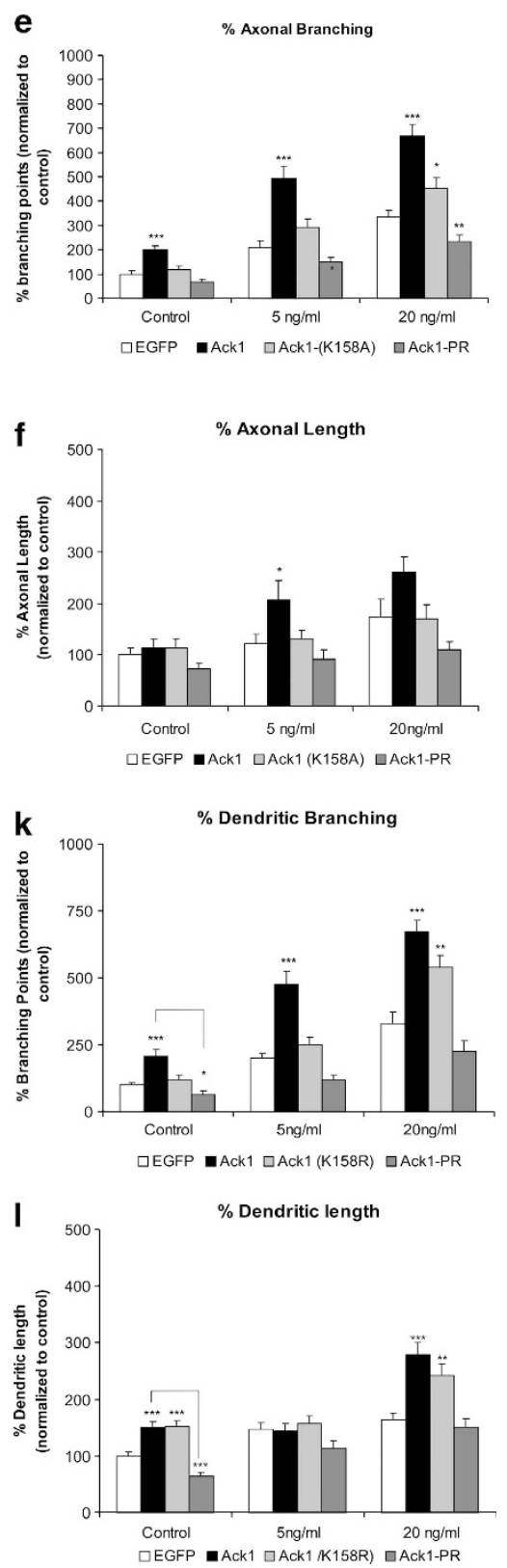

Figure 7 Ack1 enhances BDNF-dependent neuritic outgrowth and branching in hippocampal neurons. Primary hippocampal neurons were transfected with pEGFP (a and $\mathbf{g}$ ) or co-transfected with pEGFP and Ack1 (b and $\mathbf{h}$ ), Ack1 (K158A) (c and i) or the proline-rich construct ( $\mathbf{d}$ and $\mathbf{j})$, at day 4 in vitro and fixed 3 days later. Neurons were treated with a range of dosages of BDNF (control experiment, 5 or $20 \mathrm{ng} / \mathrm{ml}$ of BDNF). (a-d) Untreated cells and ( $\mathrm{g}-\mathrm{j}$ ) transfected neurons treated with $5 \mathrm{ng} / \mathrm{ml}$ of BDNF for 2 days are shown. The number of branching points of axons and dendrites of GFP-immunopositive neurons was markedly increased upon Ack1 overexpression, as also shown by quantitative analyses (e and $\mathbf{k}$ ). The length of axons ( $\mathbf{f}$ ) and dendrites (I) was also measured in GFP-immunopositive neurons. The data are represented as mean \pm S.E.M. of five separate experiments. Data were normalized to control values (pEGFP transfection). Each treatment group at 5 and $20 \mathrm{ng} / \mathrm{ml}$ was compared with its corresponding control using the $T$-test $\left({ }^{\star} P<0.01 ;{ }^{* *} P<0.05 ;{ }^{* \star} P<0.001\right)$. Scale bars: (a-d) $25 \mu \mathrm{m} ;(\mathbf{g}-\mathbf{j}) 15 \mu \mathrm{m}$

regulates the MAPK and Akt pathways; in line with this premise, overexpression of Ack1 promoted a higher activation of ERK 1/2 and Akt1 whereas endogenous Ack1 knock-down partially counteracted NGF-induced activation.

The process of neurite formation requires the assembly of cytoskeletal elements at specific membrane sites. Upon neurotrophin stimuli, Ack1 is recruited to precise localizations in the membrane edge, and it is possible that the Ack1 signaling pathway locally modifies the dynamics of actin fibers. The morphological changes that neurons undergo during their development require the re-organization of the actin cytoskeleton along with an increase in peripheral actin filaments. ${ }^{41}$ It has been well documented that the loss of stress fibers results in cell membrane protrusions and neuronal differentiation. ${ }^{42,43}$ Other molecules regulating actin dynamics such as Rho kinase have been demonstrated to 

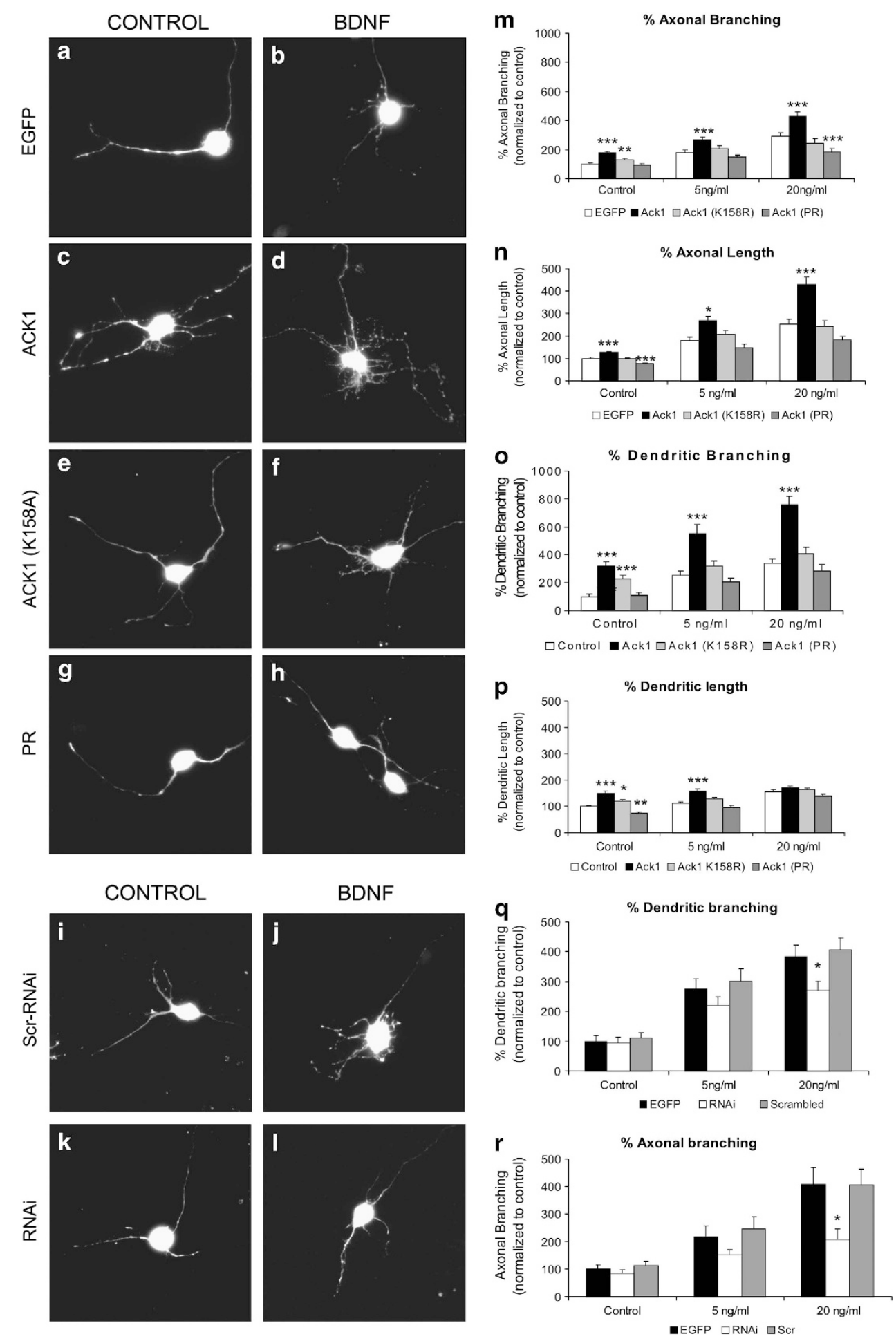

Figure 8 Ack1 promotes BDNF-induced arborization in EGL neurons. (a-h) Cerebellar granule neurons were transfected with pEGFP (a and $\mathbf{b})$ or co-transfected with pEGFP and Ack1 (c and d), Ack1 (K158A) (e and f) or the proline-rich construct ( $\mathbf{g}$ and $\mathbf{h})$, at day 1 in vitro and fixed $48 \mathrm{~h} \mathrm{later.} \mathrm{Neurons} \mathrm{were} \mathrm{treated} \mathrm{with} \mathrm{a} \mathrm{range} \mathrm{of} \mathrm{dosages} \mathrm{of}$ $\operatorname{BDNF}(\mathbf{a}, \mathbf{c}, \mathbf{e}$, and $\mathbf{g}$, control experiment; $\mathbf{b}, \mathbf{d}, \mathbf{f}$, and $\mathbf{h}, 5 \mathrm{ng} / \mathrm{ml}$ of BDNF). The number of branching of GFP-immunopositive neurons was markedly increased upon Ack1 overexpression, as also shown by quantitative analyses ( $\mathbf{m}$ and $\mathbf{~})$. ( $\mathbf{n}$ and $\mathbf{p}$ ) Quantification of axonal and dendritic length. (i-l) Cerebellar granule neurons were transfected

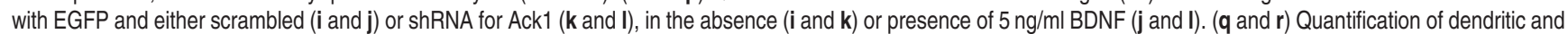
axonal branching. The mean data \pm S.E.M. of five separate experiments are shown. Data were normalized to control values ( $p E G F P$ transfection). Each treatment group at 5 and $20 \mathrm{ng} / \mathrm{ml}$ was compared with its corresponding control using $T$-test $\left({ }^{\star} P<0.01 ;{ }^{* \star} P<0.05 ;{ }^{* \star \star} P<0.001\right)$. Scale bars: $20 \mu \mathrm{m}$ 
have a role in dendritic dynamics; ${ }^{44}$ a functional connection between Ack1 and Rho kinase cannot be excluded although no data are available at present from the literature.

Our data also provide evidence that Ack1 promotes neurite branching and extension in PC12 cells and hippocampal and granule cell neurons even without the addition of NGF/BDNF. We thus propose that Ack1 has two independent, though interconnected, roles: by itself it would promote axonal and dendritic outgrowth, even independently of neurotrophins, and would also be downstream of Trk receptor activation. The receptor/signaling pathways and cascades that activate Ack1 protein in neurons to mediate neurite branching and extension in a neurotrophin-independent way remain to be determined.

In summary, the present study indicates that the tyrosine kinase Ack1 is a novel component of the neurotrophin signaling pathway as well as a protein that regulates neural extension and branching. In mature neurons, neurotrophin signaling is also required for synaptic plasticity-related events, and actin is a major cytoskeletal component required for dendritic spine morphological plasticity. ${ }^{7,45-49}$ Ack1 localizes to both the pre- and the post-synaptic compartments. Thus, our data linking Ack1 to neurotrophin signaling together with the observation that its expression is regulated by neuronal activity $^{25}$ suggest that Ack1 may exert a further potential role in synaptogenesis and synaptic plasticity events that would be addressed in future work.

\section{Materials and Methods \\ Animals. OF1 embryos and postnatal mice (Charles River, Lyon, France) were used in this study. The mating day was considered as embryonic day $0(E 0)$ and the day of birth postnatal day 0 (PO). All experimental procedures with mice were performed in accordance with the guidelines approved by the Spanish Ministry of Science and Technology and following the European Community Council Directive} 86/609 EEC.

Reagents. NGF was purchased from Peprotech Inc., (Neully sur Seine, France) and BDNF and NT-3 were obtained from Promega (Madison, WI, USA).

Polyclonal antibody against Ack1 (384) was generated using the GST fusion proteins corresponding to the proline-rich region (amino acids 502-1008), as described elsewhere. ${ }^{25}$ Monoclonal antibodies against Ack1 were generated by the company Leitat (Barcelona, Spain). As antigen, the same GST fusion protein encoding amino acids 502-1008 of Ack1 used for polyclonal antibodies was utilized. After injections of mice with the purified protein, standard procedures were followed to obtain the antibodies. ${ }^{50}$

Antibodies against phosphotyrosine (clone 4G10), TrkA, TrkB and TrkC were from Millipore (Billerica, MA, USA). A second anti-Trk A antibody was from Santa Cruz Biotechnology (Santa Cruz, CA, USA). Anti-pan-Trk $(\alpha-203)$ has been described previously. ${ }^{51}$ Anti-phospho-p42/p44 MAP kinase (Thr202/Tyr204) and anti-phospho-Akt (Ser 473) antibodies were from Cell Signaling Technology (Danvers, MA, USA). Anti pan-ERK was obtained from BD Transduction Laboratories (San Diego, CA, USA). The antibody against Akt1 (C-20) was purchased from Santa Cruz Biotechnology. Anti-GFP was from Molecular Probes/ Invitrogen (Carlsbad, CA, USA) and from Sigma (St. Louis, MO, USA), anti-actin from Chemicon (Temecula, CA, USA), and anti-HA (clone 3F10), anti-tubulin (clone KMX-1), and anti-myc (clone 9E10) from Roche (Basel, Switzerland).

Phalloidin-FITC and Hoechst (Bisbenzimide) were purchased from Sigma-Aldrich (St. Louis, MO, USA). Alexa Fluor 488 goat anti-mouse immunoglobulin G ( $\operatorname{lgG}$ ) $(\mathrm{H}+\mathrm{L})$ and Alexa Fluor 568 goat anti-rabbit immunoglobulin $\mathrm{G}(\mathrm{lg} G)(\mathrm{H}+\mathrm{L})$ were from Molecular Probes/Invitrogen. The goat anti-mouse-HRP, the swine anti-rabbitHRP, and rabbit anti-goat-HRP secondary antibodies used in the western blot were purchased from Dako (Glostrup, Denmark). Goat anti-rabbit-HRP was from Sigma. Protein G-Sepharose 4B Fast Flow was purchased from Sigma and GlutathioneSepharose $4 B$ beads from Amersham (Little Chalfont, UK). The inhibitors PD 98059,LY 294002, PP2, and PP3 used in the experiments were supplied by Calbiochem (San Diego, CA, USA).
cDNA constructs. The full-length Ack1 (Ack1-FL) tagged at the aminoterminus with $\mathrm{HA}$, and the proline-rich-GST constructs were generated as described previously. ${ }^{25}$ The Ack1 kinase-defective mutant (K158A) was generated by PCR-directed mutagenesis. Primer sequences: forward $\left(5^{\prime}-3^{\prime}\right)$ GTGAGTGT GGCCGTGGCATGCCTGAAACCT, reverse $\left(5^{\prime}-3^{\prime}\right)$ AGGTTTCAGGCATGCCACG GCCACACTCAC, the underlined codons correspond to the mutation introduced. The mutated sequence was confirmed by DNA sequencing. To knock-down endogenous Ack1, we used Sigma Mission Lentiviral Packaging shRNA technology, following the manufacturer's instructions (Sigma). Five shRNA lentiviral constructs either against Ack1 (clone ID NM_005781.4) or control RNA (scrambled) were used to generate stable PC12 cell lines. After puromycin selection, we tested the efficiency of each construct by western blot. GFP lentiviral plasmid was used as a positive transfection control.

Cell culture and transfection. HEK 293T cells were cultured in Dulbecco's Modified Eagle's Medium (DMEM) with 10\% heat-inactivated fetal bovine serum (FBS), $20 \mathrm{U} / \mathrm{ml}$ penicillin, and $20 \mu \mathrm{g} / \mathrm{ml}$ streptomycin. PC12 cells were grown on the same DMEM supplemented with $10 \%$ heat-inactivated FBS and $5 \%$ heatinactivated horse serum (HS), $20 \mathrm{U} / \mathrm{ml}$ penicillin and $20 \mu \mathrm{g} / \mathrm{ml}$ streptomycin. All cells were maintained in $5 \% \mathrm{CO}_{2}$ at $37^{\circ} \mathrm{C}$. For transfection, the cells were cultured overnight to $40-60 \%$ confluence. Transfection was performed with the Lipofectamine Plus transfection reagent, following the manufacturer's instructions (Invitrogen). For immunoprecipitation and pull-down assays, $4 \mu \mathrm{g}$ of DNA was used in $90-\mathrm{mm}$ culture plates. PC12 cells were transfected with the desired plasmid using Lipofectamine 2000 reagent (Invitrogen), following the manufacturer's instructions.

Western blot and immunoprecipitation assays. Embryonic, postnatal and adult brains, as well as primary cultures, PC12 cells and transiently transfected HEK 293T cells were collected in lysis buffer: $50 \mathrm{mM}$ HEPES (4-2 hydroxyethyl-1-piperazine ethansultanic acid), pH 7.5, $150 \mathrm{mM}$ sodium chloride, $1.5 \mathrm{mM}$ magnesium chloride, $1 \mathrm{mM}$ EGTA (ethylene glycol-bis (2-aminoethylether)- $N, N, N^{\prime}, N$-tetraacetic acid), 10\% glycerol, and 1\% Triton $\mathrm{X}-100$, containing a protease inhibitor cocktail (Roche) and phosphatase inhibitors ( $10 \mathrm{mM}$ tetra-sodium pyrophosphate, $200 \mu \mathrm{M}$ sodium orthovanadate and $10 \mathrm{mM}$ sodium fluoride). Cells were then centrifuged at 13000 r.p.m. for $20 \mathrm{~min}$ and the pellet was discarded. The supernatant was analyzed either by western blot or subjected to immunoprecipitation. For tissue extracts, samples were weighed and five volumes of lysis buffer were added. The mixture was processed using a Polytron homogenizer and insoluble material was removed by centrifugation at 13000 r.p.m. for 20 min. For immunoprecipitation experiments, antibodies were added to $500 \mu \mathrm{g}$ of total protein of cell lysates or tissue extracts, which were then incubated for $3 \mathrm{~h}$ at $4^{\circ} \mathrm{C}$. As a control, a rabbit or mouse antibody (IgG) respectively was used under the same conditions. Sepharose coupled to protein $\mathrm{G}$ (Sigma) was then added and the suspension was incubated for $2 \mathrm{~h}$ at $4^{\circ} \mathrm{C}$. After this period, Sepharose beads were washed with lysis buffer five times, then suspended in $50 \mu$ lof Lysis buffer and $25 \mu$ of Laemmli loading buffer ( $25 \mathrm{mM}$ Tris $\mathrm{pH} 6.5,0.17 \mathrm{mM} \beta$-mercaptoethanol, $2 \%$ SDS, $10 \%$ glycerol, and $0.0025 \%$ bromophenol blue) were added and the samples were analyzed by SDS-PAGE. ${ }^{52}$

Samples were run in an $8 \%$ polyacrylamide gel at $120 \mathrm{~V}$, after which they were transferred onto a nitrocellulose membrane in $120 \mathrm{mM}$ glycine, $125 \mathrm{mM}$ Tris, pH 8.5 and $20 \%$ methanol. Filters were then saturated in $3 \%$ BSA in TBS and incubated with the antibodies. Detection was performed using the ECL system from Amersham. For quantification, films were scanned and then subjected to band densitometry and quantification (Bio-Rad, Hercules, CA, USA).

Kinase assay. To determine Ack1 activity, we used the Tyrosine Kinase Assay Kit (Cat no. 17-315) from Upstate Cell Signaling Solutions/Millipore (Billerica, MA, USA) following the manufacturer's instructions. In brief, we used transfected HEK 293T cells, PC12 cells, or hippocampal neurons, plated onto 35-mm cell culture dishes (Nunc, Roskilde, Denmark) and stimulated with NGF, BDNF, or NT-3 for a range of times $(0,5,15,30$, and $60 \mathrm{~min})$ and in some cases treated with PI3K inhibitor (LY294002, $50 \mu \mathrm{M}$ ) or with MERK inhibitor (PD 98059, $50 \mu \mathrm{M})$; the cells were lysed and immunoprecipitated with anti-Ack1 antibodies as described above. The immunoprecipitated Ack1 was assayed in a 30-min reaction in $1 \mathrm{mM}$ sodium orthovanadate, $5 \mu \mathrm{g} / \mathrm{ml} 1 \mathrm{mM}$, Poly (Glu4-Tyr) peptide, conjugated biotin, $20 \mathrm{mM}$ Tris- $\mathrm{HCl} \mathrm{pH} \mathrm{7.4,} 10 \mathrm{mM} \mathrm{MgCl}, 1 \mathrm{mM} \mathrm{MnCl} 2,1 \mathrm{mM}$ dithiothreitol and $200 \mu \mathrm{M}$ ATP. The reaction was stopped by heat inactivation $\left(5 \mathrm{~min}\right.$ at $\left.95^{\circ} \mathrm{C}\right)$ and transferred to ELISA plates. Phosphotyrosine was detected with conjugated 
anti-phosphotyrosine antibody-HRP and tetramethylbenzidine reagent coupled to hydrogen peroxide in citrate buffer, following the manufacturer's instructions. Quantification was done by spectrophotometric absorbance measurements at $450 \mathrm{~nm}$.

PC12 differentiation. PC12 cells were grown and maintained as described above. Differentiation was stimulated with $50 \mathrm{ng} / \mathrm{ml}$ of NGF in DMEM containing $0.5 \%$ NHS and allowed to proceed for up to 10 days.

Generation of shRNA-resistant Ack1 and rescue experiments. An Ack1 resistant to shRNA was generated by site-directed mutagenesis with QuickChange XL Site-Directed Mutagenesis Kit (Stratagene, Agilent Technologies, Santa Clara, CA, USA), following the manufacturer's instructions. The following primers were used to amplify Ack1: Forward $\left(5^{\prime}-3^{\prime}\right)$ : CATGTGCAAACG CAAAAGTTGGATGTCCAAGGTGTTCAGTG and Reverse $\left(5^{\prime}-3^{\prime}\right)$ : CACTGAAC ACCTTGGACATCCAACTTTTGCGTTTGCACATG.

For rescue experiments, Ack-RNAi-PC12 cells were grown and maintained as above described. For our purposes, 20000 cells were seeded in $35-\mathrm{mm}$ plates and grown overnight in complete medium. The next day, cells were transfected with Lipofectamine 2000 (Invitrogen) and $0.8 \mu \mathrm{g}$ of DNA per plate and left 1 more day in complete medium. The following day, cells were deprived overnight in DMEM containing $0.5 \%$ normal $\mathrm{HS}$, and then differentiation was stimulated with $50 \mathrm{ng} / \mathrm{ml}$ NGF in the same medium and allowed to proceed for up to 10 days. During this time, photographs of random fields were taken with an inverted microscope Nikon TE200 (Izasa, Barcelona, Spain) equipped with optics coupled to a digital camera. Neurite outgrowth was quantified using ImageJ software (Wayne Rasband, $\mathrm{NIH}$ ).

Cell viability. Several lines of PC12 cells stably expressing Ack1, RNAi-1, RNAi2 , or Scr were used. To determine cell viability, we used the WST-1 cell viability assay from Roche (Mannheim, Germany), following the manufacturer's instructions.

Paraformaldehyde fixation, immunofluorescent staining, and F-actin staining. Cells were fixed with $4 \%$ paraformaldehyde in PBS (pH 7.4) for $30 \mathrm{~min}$ or frozen methanol for $15 \mathrm{~min}$. For immunocytochemistry, cells were permeabilized and blocked with $0.1 \%$ Triton X-100 (Sigma) and 10\% normal goat serum in PBS for $1 \mathrm{~h}$, washed with PBS and incubated with primary antibodies or Phalloidin-FITC diluted in PBS (polyclonal Ack1 1: 100; monoclonal Ack1 1:25; anti-GFP 1:1000; monoclonal NGF 1:100; Trk A 1/100, pan-Trk 203 antibody $1: 1000$; $p$-ERK 1:1000, Phalloidin-FITC $1: 500$ ) for $2 \mathrm{~h}$. After being washed with PBS, cells were incubated with either secondary antibodies goat anti-mouse-488 and goat anti-rabbit-568 or goat anti-rabbit-488 (Alexa Fluor, Invitrogen) for $1 \mathrm{~h}$. Coverslips were washed with PBS and mounted on glass slides. All procedures were carried out at room temperature.

Neuronal primary cultures and transfection. For hippocampal primary cultures, E16 mouse brains were dissected and dissociated, as described before. ${ }^{25}$ Primary hippocampal neurons were seeded $\left(3 \times 10^{6}\right.$ cells per $35-\mathrm{mm}$ dish for biochemical analyses and 100000 cells per coverslip in transfection experiments) on dishes or coverslips pre-coated with poly-D-lysine (Sigma). Cerebellar granule cell cultures were performed as described by Hatten and collaborators. ${ }^{53}$ After granule cell purification, we plated 150000 neurons plated on poly-L-lysine (Sigma) pre-coated coverslips. Neuron transfections were performed at day 4 (hippocampal neurons) and day 1 (granule cells), following the instructions of the manufacturer, using $1 \mu \mathrm{l}$ of Lipofectamine 2000 (Invitrogen) and $1 \mu \mathrm{g}$ of DNA per well of a 24-well plate. Co-transfections of Ack1 constructs and pEGFP plasmids were performed at a proportion of $5: 1$ (Ack1:EGFP). For RNAi induction, we co-transfected neurons with EGFP- and shRNA-containing plasmids from the MISSION shRNA library corresponding to Ack1 (NM_005781.4) in the above-mentioned proportion 5:1 (shRNA:EGFP).

Quantification of neurite outgrowth and branching. In PC12 differentiation experiments, photographs of random fields were taken 1-10 days after NGF treatments using an inverted microscope Nikon TE200 equipped with optics coupled to a digital camera. Neurite outgrowth was measured using the AnalySIS software (Soft Imaging System, Munster, Germany) and ImageJ software. In each experiment, a minimum of 150 cells were measured per condition. Cells were considered as differentiated when they presented one neurite 1.5 times longer than the soma.

Labeled EGL or hippocampal neurons were visualized and digitally acquired in a Nikon TE1000 microscope (Nikon Instruments, Izasa). For each condition studied, we selected about 50-60 cells. Neuritic arbors were traced and analyzed with AnalySIS software or by direct counting of the branching points and other topological parameters. Co-localization experiments were performed using a Leica TCS SP2 AOBS confocal microscope. For statistical analyses, we used Excel 2003.

Determination of Ras activity. To determine Ras activity, we used a Rasactivation assay kit from Millipore. In brief, the assay consists of pulling down active Ras (Ras-GTP) with GST-Raf1 RBD coupled to glutathione-agarose, followed by Ras detection using a pan-Ras antibody.

\section{Conflict of Interest}

The authors declare no conflict of interest.

Acknowledgements. We thank Dr. Daniel Cifuentes for his advice and support with lentiviral infections and Raquel Tomé for her help with the proline-rich construct. We thank Dr. PA Barker and Dr. Rosalind A Segal for the Trk constructs. Thanks also go to Dr. Patrick Morcillo for critical reading of the manuscript, Dr. Rosa Andrés, Oriol Ros, and Eva M Pastor for technical assistance; and Tanya Yates for editorial assistance. This work was supported by grants from Spanish Ministry of Health ISCIII PI042280, PI070942, and PI10/01750 to JMU, the Spanish Ministry of Science (MICINN) BFU2008-0743, and CIBERNED (Centro de Investigación Biomédica en Red para Enfermedades Neurodegenerativas) CB06/05/0098 to ES and JAR, and SAF2010-19953 and CIBERNED CB06/05/0042 to JXC. MMM. was recipient of a fellowship from University of Barcelona for postgraduate student (APIF program) RSM was supported by a Juan de la Cierva grant.

1. Barnes AP, Polleux F. Establishment of axon-dendrite polarity in developing neurons. Annu Rev Neurosci 2009; 32: 347-381.

2. Gao FB. Molecular and cellular mechanisms of dendritic morphogenesis. Curr Opin Neurobiol 2007; 17: 525-532.

3. Jan YN, Jan LY. The control of dendrite development. Neuron 2003; 40: 229-242.

4. Miller FD, Kaplan DR. On Trk for retrograde signaling. Neuron 2001; 32: 767-770.

5. Whitford KL, Dijkhuizen P, Polleux F, Ghosh A. Molecular control of cortical dendrite development. Annu Rev Neurosci 2002; 25: 127-149.

6. Ernsberger $U$. Role of neurotrophin signalling in the differentiation of neurons from dorsal root ganglia and sympathetic ganglia. Cell Tissu Res 2009; 336: 349-384.

7. McAllister AK. Neurotrophins and neuronal differentiation in the central nervous system. Cell Mol Life Sci 2001; 58: 1054-1060.

8. Tucker KL, Meyer M, Barde YA. Neurotrophins are required for nerve growth during development. Nat Neurosci 2001; 4: 29-37.

9. Huang EJ, Reichardt LF. Trk receptors: roles in neuronal signal transduction. Annu Rev Biochem 2003; 72: 609-642.

10. Diarra A, Geetha T, Potter P, Babu JR. Signaling of the neurotrophin receptor p75 in relation to Alzheimer's disease. Biochem Biophys Res Commun 2009; 390: 352-356

11. Minichiello L. TrkB signalling pathways in LTP and learning. Nat Rev Neurosci 2009; 10 : 850-860; Review.

12. Manser E, Leung T, Salihuddin H, Tan L, Lim L. A non-receptor tyrosine kinase that inhibits the GTPase activity of p21cdc42. Nature 1993; 363: 364-367.

13. Satoh T, Kato J, Nishida K, Kaziro Y. Tyrosine phosphorylation of ACK in response to temperature shift-down, hyperosmotic shock, and epidermal growth factor stimulation. FEBS Lett 1996; 386: 230-234.

14. Linseman DA, Heidenreich KA, Fisher SK. Stimulation of M3 muscarinic receptors induces phosphorylation of the Cdc42 effector activated Cdc42Hs-associated kinase-1 via a Fyn tyrosine kinase signaling pathway. J Biol Chem 2001; 276: 5622-5628.

15. Yang W, Lin Q, Zhao J, Guan JL, Cerione RA. The nonreceptor tyrosine kinase ACK2, a specific target for $\mathrm{Cdc} 42$ and a negative regulator of cell growth and focal adhesion complexes. J Biol Chem 2001; 276: 43987-43993.

16. Yokoyama N, Miller WT. Biochemical properties of the Cdc42-associated tyrosine kinase ACK1. Substrate specificity, autophosphorylation, and interaction with Hck. J Biol Chem 2003; 278: 47713-47723.

17. Kiyono M, Kato J, Kataoka T, Kaziro Y, Satoh T. Stimulation of Ras guanine nucleotide exchange activity of Ras-GRF1/CDC25(Mm) upon tyrosine phosphorylation by the Cdc42regulated kinase ACK1. J Biol Chem 2000; 275: 29788-29793.

18. Kato J, Kaziro Y, Satoh T. Activation of the guanine nucleotide exchange factor Dbl following ACK1-dependent tyrosine phosphorylation. Biochem Biophys Res Commun 2000; 268: 141-147.

19. Yokoyama N, Lougheed J, Miller WT. Phosphorylation of WASP by the Cdc42 associated kinase ACK1: dual hydroxyamino acid specificity in a tyrosine kinase. J Biol Chem 2005; 280: 42219-42226. 
20. Eisenmann KM, McCarthy JB, Simpson MA, Keely PJ, Guan JL, Tachibana K et al. Melanoma chondroitin sulphate proteoglycan regulates cell spreading through $\mathrm{Cdc42}$, Ack-1 and p130cas. Nat Cell Biol 1999; 1: 507-513.

21. Galisteo ML, Yang Y, Urena J, Schlessinger J. Activation of the nonreceptor proteintyrosine kinase Ack by multiple extracellular stimuli. Proc Natl Acad Sci USA 2006; 103: 9796-9801.

22. Kato-Stankiewicz J, Ueda S, Kataoka T, Kaziro Y, Satoh T. Epidermal growth factor stimulation of the ACK1/Dbl pathway in a Cdc42 and Grb2 dependent manner. Biochem Biophys Res Commun 2001; 284: 470-477.

23. Yang W, Lin Q, Guan JL, Cerione RA. Activation of the Cdc42-associated tyrosine kinase-2 (ACK-2) by cell adhesion via integrin beta1. J Biol Chem 1999; 274: 8524-8530.

24. La Torre A, del Rio JA, Soriano E, Urena JM. Expression pattern of ACK1 tyrosine kinase during brain development in the mouse. Gene Expr Patterns 2006; 6: 886-892.

25. Urena JM, La Torre A, Martinez A, Lowenstein E, Franco N, Winsky-Sommerer R et al. Expression, synaptic localization, and developmental regulation of Ack1/Pyk1, a cytoplasmic tyrosine kinase highly expressed in the developing and adult brain. $J$ Comp Neurol 2005; 490: 119-132.

26. Watson FL, Heerssen HM, Moheban DB, Lin MZ, Sauvageot CM, Bhattacharyya A et al. Rapid nuclear responses to target-derived neurotrophins require retrograde transport of ligand-receptor complex. J Neurosci 1999; 19: 7889-7900.

27. Kryl D, Yacoubian T, Haapasalo A, Castren E, Lo D, Barker PA. Subcellular localization of full-length and truncated Trk receptor isoforms in polarized neurons and epithelial cells. J Neurosci 1999; 19: 5823-5833.

28. Podlesniy P, Kichev A, Pedraza C, Saurat J, Encinas M, Perez B et al. Pro-NGF from Alzheimer's disease and normal human brain displays distinctive abilities to induce processing and nuclear translocation of intracellular domain of p75NTR and apoptosis. Am J Pathol 2006; 169: 119-131.

29. Greene LA, Tischler AS. Establishment of a noradrenergic clonal line of rat adrenal pheochromocytoma cells which respond to nerve growth factor. Proc Natl Acad Sci USA 1976; 73: 2424-2428.

30. Schmidt G, Wittinghofer A. Priming of PC12 cells for semiquantitative microinjection studies involving Ras. FEBS Lett 2000; 474: 184-188.

31. McAllister AK, Katz LC, Lo DC. Opposing roles for endogenous BDNF and NT-3 in regulating cortical dendritic growth. Neuron 1997; 18: 767-778.

32. Nguyen T, Di Giovanni S. NFAT signaling in neural development and axon growth. Int J Dev Neurosci 2008; 26: 141-145.

33. Arévalo JC, Chao MV. Axonal growth: where neurotrophins meet Wnts. Curr Opin Cell Biol 2005; 17: 112-115

34. Mott HR, Owen D, Nietlispach D, Lowe PN, Manser E, Lim L et al. Structure of the small G protein Cdc42 bound to the GTPase-binding domain of ACK. Nature 1999; 399: 384-388.

35. MacDonald JI, Verdi JM, Meakin SO. Activity-dependent interaction of the intracellular domain of rat trkA with intermediate filament proteins, the beta- 6 proteasomal subunit, Ras-GRF1, and the p162 subunit of elF3. J Mol Neurosci 1999; 13: 141-158.

36. Robinson KN, Manto K, Buchsbaum RJ, MacDonald JI, Meakin SO. Neurotrophindependent tyrosine phosphorylation of Ras guanine-releasing factor 1 and associated neurite outgrowth is dependent on the HIKE domain of TrkA. J Biol Chem 2005; 280: 225-235.
37. Heckman CA, Urban JM, Cayer M, Li Y, Boudreau N, Barnes J et al. Novel p21-activated kinase-dependent protrusions characteristically formed at the edge of transformed cells. Exp Cell Res 2004; 295: 432-447.

38. Worby C, Margolis B. Positive versus negative signaling of LET-23: regulation through the adaptor protein, SEM-5. Sci STKE 2000; 2000: pe2.

39. Shen F, Lin Q, Gu Y, Childress C, Yang W. Activated Cdc42-associated kinase 1 is a component of EGF receptor signaling complex and regulates EGF receptor degradation. Mol Biol Cell 2007; 18: 732-742.

40. Read DE, Gorman AM. Involvement of Akt in neurite outgrowth. Cell Mol Life Sci 2009; 66: 2975-2984.

41. da Silva JS, Dotti CG. Breaking the neuronal sphere: regulation of the actin cytoskeleton in neuritogenesis. Nat Rev Neurosci 2002; 3: 694-704.

42. Katoh H, Aoki J, Ichikawa A, Negishi M. p160 RhoA-binding kinase ROKalpha induces neurite retraction. J Biol Chem 1998; 273: 2489-2492.

43. Kozma R, Sarner S, Ahmed S, Lim L. Rho family GTPases and neuronal growth cone remodelling: relationship between increased complexity induced by $\mathrm{Cdc} 42 \mathrm{Hs}$, Rac1, and acetylcholine and collapse induced by RhoA and lysophosphatidic acid. Mol Cell Biol 1997; 17: 1201-1211.

44. Suo L, Lu H, Ying G, Capecchi MR, Wu Q. Protocadherin clusters and cell adhesion kinase regulate dendrite complexity through Rho GTPase. Mol Cell Biol 2012; 4: 362-376.

45. Luo L. Actin cytoskeleton regulation in neuronal morphogenesis and structural plasticity. Annu Rev Cell Dev Biol 2002; 18: 601-635.

46. Bramham CR, Wells DG. Dendritic mRNA: transport, translation and function. Nat Rev Neurosci 2007; 8: 776-789.

47. Flavell SW, Greenberg ME. Signaling mechanisms linking neuronal activity to ne expression and plasticity of the nervous system. Annu Rev Neurosci 2008; 31: 563-590.

48. Poo MM. Neurotrophins as synaptic modulators. Nat Rev Neurosci. 2001; 2: 24-32.

49. Schubert V, Da Silva JS, Dotti CG. Localized recruitment and activation of RhoA underlies dendritic spine morphology in a glutamate receptor-dependent manner. J Cell Biol 2006; 172: 453-467.

50. Harlow E, Lane D. Antibodies. A laboratory manual. Cold Spring Harbor Laboratory Ed.: New York, 1988.

51. Peiró S, Comella JX, Enrich C, Martin-Zanca D, Rocamora N. PC12 cells have caveolae that contain TrkA. J Biol Chem 2000; 275: 37846-37852.

52. Sambrook J, Fritsch EF, Maniatis T. Molecular Cloning. A Laboratory Manual. 2nd edn. Cold Spring Harbor Laboratory Press, Plainview, New York, 1989.

53. Gao WQ, Liu XL, Hatten ME. The weaver gene encodes a nonautonomous signal for CNS neuronal differentiation. Cell 1992; 68: 841-854.

cc) $\odot \odot$ Cell Death and Disease is an open-access journal published by Nature Publishing Group. This work is licensed under the Creative Commons Attribution-NonCommercial-No Derivative Works 3.0 Unported License. To view a copy of this license, visit http://creativecommons.org/licenses/by-nc-nd/3.0/

Supplementary Information accompanies this paper on Cell Death and Disease website (http://www.nature.com/cddis) 